\title{
Functional Differentiation of Mouse Visual Cortical Areas Depends upon Early Binocular Experience
}

\author{
${ }^{\circledR}$ Kirstie J. Salinas, ${ }^{1}{ }^{\circledR}$ Carey Y. L. Huh, ${ }^{1}$ Jack H. Zeitoun, ${ }^{1}$ and ${ }^{\circledR}$ Sunil P. Gandhi ${ }^{1,2}$ \\ ${ }^{1}$ Department of Neurobiology and Behavior, University of California, Irvine, Irvine, California 92697, and ${ }^{2}$ Center for the Neurobiology of Learning \\ and Memory, University of California, Irvine, Irvine, California 92697
}

The mammalian visual cortex contains multiple retinotopically defined areas that process distinct features of the visual scene. Little is known about what guides the functional differentiation of visual cortical areas during development. Recent studies in mice have revealed that visual input from the two eyes provides spatiotemporally distinct signals to primary visual cortex (V1), such that contralateral eye-dominated V1 neurons respond to higher spatial frequencies than ipsilateral eye-dominated neurons. To test whether binocular visual input drives the differentiation of visual cortical areas, we used two-photon calcium imaging to characterize the effects of juvenile monocular deprivation (MD) on the responses of neurons in V1 and two higher visual areas, LM (lateromedial) and PM (posteromedial). In adult mice of either sex, we find that MD prevents the emergence of distinct spatiotemporal tuning in V1, LM, and PM. We also find that, within each of these areas, MD reorganizes the distinct spatiotemporal tuning properties driven by the two eyes. Moreover, we find a relationship between speed tuning and ocular dominance in all three areas that MD preferentially disrupts in V1, but not in LM or PM. Together, these results reveal that balanced binocular vision during development is essential for driving the functional differentiation of visual cortical areas. The higher visual areas of mouse visual cortex may provide a useful platform for investigating the experience-dependent mechanisms that set up the specialized processing within neocortical areas during postnatal development.

Key words: critical period; functional differentiation; higher visual areas; monocular deprivation; ocular dominance plasticity; visual cortex

\section{Significance Statement}

Little is known about the factors guiding the emergence of functionally distinct areas in the brain. Using in vivo $\mathrm{Ca}^{2+}$ imaging, we recorded visually evoked activity from cells in V1 and higher visual areas LM (lateromedial) and PM (posteromedial) of mice. Neurons in these areas normally display distinct spatiotemporal tuning properties. We found that depriving one eye of normal input during development prevents the functional differentiation of visual areas. Deprivation did not disrupt the degree of speed tuning, a property thought to emerge in higher visual areas. Thus, some properties of visual cortical neurons are shaped by binocular experience, while others are resistant. Our study uncovers the fundamental role of binocular experience in the formation of distinct areas in visual cortex.

\section{Introduction}

The mammalian visual system has been described classically as a hierarchically organized system that transforms different aspects

\footnotetext{
Received Mar. 6, 2020; revised Dec. 15, 2020; accepted Dec. 17, 2020.

Author contributions: K.J.S., C.Y.L.H., and S.P.G. designed research; K.J.S. and C.Y.L.H. performed research; J.H.Z. contributed unpublished analytic tools; K.J.S. analyzed data; K.J.S., C.Y.L.H., and S.P.G. wrote the paper.

This work was supported by the National Institutes of Health (NIH) Director's New Innovator Award (DP2EY-024504-01), NIH Grant 1R01-EY-029490-01A1, a Searle Scholars Award, and a Klingenstein Fellowship to S.P.G. K.J.S. was supported by a National Science Foundation Graduate Research Fellowship (DGE-1321846). C.Y.L.H. was supported by Knights Templar Foundation Grants 207834 and 210510). We thank C. Niell for providing the tet0-GCaMP6 mice.

The authors declare no competing financial interests.

Correspondence should be addressed to Sunil P. Gandhi at sunil.gandhi@uci.edu.

https://doi.org/10.1523/JNEUROSCI.0548-20.2020

Copyright $\odot 2021$ the authors
}

of the retinal image along parallel streams for simultaneous processing (Felleman and Van Essen, 1991; Nassi and Callaway, 2009). The differentiation of higher visual areas (HVAs) is crucial for the encoding of higher-level visual features, such as global form and motion processing (Merigan and Maunsell, 1993). The developmental mechanisms that guide the functional differentiation of HVAs are largely unknown. A recent study using intrinsic signal optical imaging (ISOI) revealed that mouse visual areas become functionally differentiated after eye opening (Murakami et al., 2017), during a time window similar to that of the critical period for ocular dominance plasticity. Also using ISOI, a second study showed that different visual areas are sensitive to dark rearing during distinct developmental timelines (Smith et al., 2017). It remains unexplored, however, what role visual experience plays in driving the functional differentiation of visual cortical areas. 
In addition to the dearth of understanding about visual area differentiation, little is known about the mechanisms that shape the specialization of functional response properties within areas. Primate primary visual cortex (V1) cells vary widely in their spatiotemporal tuning and ocular dominance properties (Livingstone and Hubel, 1987; Nassi and Callaway, 2009). In mice, subpopulations of V1 cells have unique spatiotemporal selectivity (Gao et al., 2010; Ji et al., 2015), similar to the specialization of higher visual areas in other species that can be grouped into putative visual streams (Andermann et al., 2011; Marshel et al., 2011; Wang et al., 2011, 2012; Roth et al., 2012; Murakami et al., 2017; Smith et al., 2017). To understand the development of functional differentiation of visual cortical areas, it is critical to characterize the tuning properties of individual cells within clearly defined areal boundaries.

We recently discovered in mouse V1 that neurons with common ocular dominance properties also share similar spatial frequency selectivity (Salinas et al., 2017). This coupling of ocular dominance with spatial frequency tuning has implications for the functional differentiation of visual areas whose spatiotemporal properties are distinct from one another. We wondered whether the link between ocular dominance and spatiotemporal selectivity extends to cellular responses in HVAs. Given the distinct spatial frequency input provided by the two eyes to V1, we hypothesized that monocular deprivation (MD) would lead to a disruption in the functional differentiation of visual cortical areas.

Using wide-field calcium imaging to precisely define area boundaries together with two-photon calcium imaging to resolve single-cell responses, we recorded from thousands of excitatory neurons in layer 2/3 (L2/3) of mouse areas V1, LM, and PM to determine their spatiotemporal and eye-specific response properties. We find that 2 weeks of MD during the ocular dominance critical period disrupts the functional differentiation of speed preferences in these visual cortical areas into adulthood. MD also reorganizes the relationship between ocular dominance properties and speed-tuning preferences in these areas. Interestingly, in control mice, we find that the degree of speed tuning is greater in LM and PM than in V1, consistent with the idea that speed tuning is a functional property that arises in higher visual areas. In all three areas, ocular dominance interacts with speed tuning such that contralateral eye-dominated neurons display more speed tuning than ipsilateral eye-dominated neurons. Contrary to the prediction of a simple hierarchical model, we find that MD perturbs the relationship between ocular dominance and degree of speed tuning in V1 but not in higher areas. Altogether, our results show that early binocular vision is important for areal differentiation of functional visual properties in mouse visual cortex.

\section{Materials and Methods}

Animals

All protocols and procedures followed the guidelines of the Animal Care and Use Committee at the University of California, Irvine. To image visually-evoked activity in layer $2 / 3$ excitatory neurons in multiple areas, a CAMK2a-tTa driver line (RRID:IMSR_JAX: 007004) was crossed with a line expressing the calcium indicator GCaMP6s under the control of the tetracycline-responsive regulatory element (tetO; RRID:IMSR_JAX:024742; Wekselblatt et al., 2016). The founder line was heterozygous for both transgenes and was maintained by breeding with wild-type C57BL/6 mice (RRID: IMSR_CRL:642). Mice of either sex were weaned at postnatal day 18 (P18) to P21 and cohoused with one or more littermate until the day of window implantation (P73 to P200). In all experiments, both female and male mice were used. Mice were kept on a $12 \mathrm{~h}$ light/ dark cycle and housed in conventional mouse cages.

\section{Monocular deprivation}

For MD experiments, one eye was sutured (either right or left) at P19 and the sutures were monitored for 2 weeks (Davis et al., 2015). Under isoflurane anesthesia ( $2 \%$ for induction, $1 \%$ for maintenance), the nondeprived eye was covered with ophthalmic ointment while the other eye was sutured closed with two or three mattress sutures (7-0 silk, Ethicon). If the eye opened at any time before the 2 week window, the mouse was removed from the experiment. Eyes were opened after 2 weeks and inspected for any damage under a microscope. Some mice were deprived in the eye contralateral to the recorded hemisphere [contralateral eye MD (CMD)], while others were deprived in the ipsilateral eye [ipsilateral eye MD (IMD)]. In total, we gathered data from eight control mice (No MD), six CMD mice, and four IMD mice.

\section{Cranial window implantation}

Mice were anesthetized with isoflurane in $\mathrm{O}_{2}$ (2\% for induction, $1-1.5 \%$ for maintenance). Headplate attachment and craniotomy were performed in one surgery. Carprofen $(5 \mathrm{mg} / \mathrm{kg}$, s.c.) and topical lidocaine $(2 \%, 20 \mathrm{mg} / \mathrm{ml})$ were administered to provide analgesia. Dexamethasone was administered $4-8 \mathrm{~h}$ before surgery $(4.8 \mathrm{mg} / \mathrm{kg}$, i.m.). Atropine $(0.15 \mathrm{mg} / \mathrm{kg}$, s.c.) was administered to reduce secretions and aid in respiration. To attach custom-printed ABS headplates, the skull was cleared of connective tissue. A thin layer of Vetbond was applied to the skull, and the headplate was attached using dental acrylic at an angle parallel to the site of imaging $\left(\sim 20^{\circ}\right.$ from horizontal). A craniotomy (diameter, $5 \mathrm{~mm}$ ) was performed over either the left or right hemisphere using previously described methods (Salinas et al., 2017). A $5 \mathrm{~mm}$ glass coverslip (World Precision Instruments) was placed over the exposed brain and sealed with Vetbond and black dental acrylic (Lang Dental). Sterile eye ointment (Rugby) was used to protect the eyes. Body temperature was maintained at $37^{\circ} \mathrm{C}$ using a heating pad under feedback control from a rectal thermoprobe. Mice recovered on a warm heating pad following surgery and were provided with lactated Ringer's solution for hydration. Mice were given daily injections of carprofen $(5 \mathrm{mg} / \mathrm{kg}$, s.c.) for at least $2 \mathrm{~d}$ postsurgery.

\section{Visual area mapping}

Mapping of the visual areas was performed at least 1 week after window installation using wide-field imaging of GCaMP6s (Wekselblatt et al., 2016; Zhuang et al., 2017). Wide-field fluorescence images were acquired using a SciMedia THT macroscope (Leica PlanApo 1.0×; imaging area, $6.5 \times 6.5 \mathrm{~mm}$ ) equipped with an Andor Zyla sCMOS camera. The surface vasculature and GCaMP6s signal was visualized using a blue $465 \mathrm{~nm}$ LED (LEX2). The camera was focused $\sim 600 \mu \mathrm{m}$ beneath the surface. Image acquisition and visual stimulus presentation was controlled by custom-written software in Python using the PsychoPy 1.8 library.

\section{Visual area mapping stimuli}

Visual area mapping was performed following previously published procedures (Salinas et al., 2017). Briefly, mice were shown a $20^{\circ}$ wide visual noise stimulus that swept periodically every $10 \mathrm{~s}$ in each of the four cardinal directions. The sweeping visual stimulus was created by multiplying a band limited $(<0.5 \mathrm{cyc} / \mathrm{deg} ;>2 \mathrm{~Hz})$, binarized spatiotemporal noise movie with a one-dimensional spatial mask $\left(20^{\circ}\right)$ that was phase modulated at $0.1 \mathrm{~Hz}$. A gamma-corrected monitor (54 inch LED TV; model 55LB5900, LG) with maximum luminance of $30 \mathrm{~cd} / \mathrm{m}^{2}$ was placed $25 \mathrm{~cm}$ from the contralateral eye and angled at $\sim 30^{\circ}$ from the long axis of the animal. The stimulus was spherically corrected to cover a $140^{\circ}$ visual angle in elevation and $120^{\circ}$ in azimuth. The stimulus was presented to the contralateral eye for $5 \mathrm{~min}$ for each direction. To confirm the location of the binocular zone, we also presented the sweeping, binarized noise stimulus confined to the central $30^{\circ}$ of visual azimuth.

\section{Analysis for mapping stimuli}

Retinotopic maps of azimuth and elevation were used to generate a visual field sign map (Sereno et al., 1994; Garrett et al., 2014) to 
designate borders between visual areas. The visual field sign map as used to quantify area size with the area tool using ImageJ. Recordings were directed as close to the central visual field as possible in each area, although biases exist in the retinotopic organization of areas LM and PM (Zhuang et al., 2017). Recordings from binocular V1 were confined to regions adjacent to the intersection of the horizontal and vertical meridians at the border of V1 and LM. Recordings from area LM were restricted to the anterior portion of LM, while recordings from PM were performed in both the anterior portion, covering the relatively lower elevation and posterior portion of PM, covering relatively higher elevation. Although we did notice an enhancement for spatial frequency tuning in higher elevations of PM (posterior PM), we decided to pool all responses to increase statistical power.

\section{Two-photon calcium imaging}

Cellular imaging was performed in awake, head-fixed mice that were acclimated to the setup. Fluorescence was gathered with a resonant two-photon microscope (Neurolabware) with a 900-920 nm excitation laser (Mai Tai HP, Spectra-Physics). Emissions were filtered using a $510 / 84 \mathrm{~nm}$ BrightLine bandpass filter (Semrock). A $16 \times$ (numerical aperture 0.8 ; Nikon) water-immersion lens was used. Image sequences typically covered a field of $\sim 700 \times 500 \mu \mathrm{m}$ for cell recordings in V1 and LM or $\sim 500 \times 400 \mu \mathrm{m}$ for cell recordings in PM and were acquired at 7.7 or $12 \mathrm{~Hz}$ (1024 or 660 lines) using Scanbox acquisition software (Neurolabware) at a depth of $200-250 \mu \mathrm{m}$ below the pia.

\section{Two-photon visual stimuli}

Visual stimuli were generated by custom-written Python code using the PsychoPy 1.8 library. An Acer V193 gamma-corrected monitor (54 inch LED TV; model 55LB5900, LG; $60 \mathrm{~Hz}$ refresh rate, $30 \mathrm{~cd} / \mathrm{m}^{2}$ ) was used. For the speed-tuning experiment, full-field drifting sinusoidal gratings were presented at four orientations $(0,90,180$, and 270$)$, five spatial frequencies $(0.03,0.06$, $0.12,0.24$, and $0.48 \mathrm{cyc} / \mathrm{deg})$, and four temporal frequencies $(1,2,4$, and $8 \mathrm{~Hz}$ ). The visual stimulus was spherically corrected. In addition to the 80 grating stimuli (four directions $*$ five spatial frequencies $*$ four temporal frequencies), we also showed a blank condition and a condition in which the whole monitor flickered at $2 \mathrm{~Hz}$ (full-field flicker, FF). The 82 total stimulus conditions were presented in a random order for each of the eight repetitions. For each trial, the stimulus was presented for $2 \mathrm{~s}$, followed by $2 \mathrm{~s}$ of gray screen. The visual stimulus was presented either first to the ipsilateral or the contralateral eye using an occluder to block presentation to the other eye.

\section{Data analysis}

Cellular responses. Custom-written Python routines were used to remove motion artifacts, identify cell ROIs, extract calcium fluorescence traces, and perform analyses. First, we implemented motion correction by using an efficient algorithm that corrects for translational artifacts by minimizing the Euclidean distance between frames and a template image using a Fourier transform approach (Dubbs et al., 2016). To identify the region of pixels associated with distinct neuronal cell bodies, we used the maximum intensity projection of the images. Only cell bodies that could be visually identified throughout both contralateral and ipsilateral eye viewing condition recordings were included in the analysis. The fluorescence signal of a cell body at time $t$ was determined as $F_{\text {cell }}(t)=F_{\text {soma }}(t)-(R$ (neuropil contamination ratio $) \times$ $\left.F_{\text {neuropi }} l(t)\right)$ (Kerlin et al., 2010; Chen et al., 2013). $R$ was empirically determined to be 0.7 by comparing the intensity of GCaMP6s signal in the blood vessels to the intensity in the neuropil across recordings. The neuropil signal $F_{\text {neuropil }}(t)$ of each cell was measured by averaging the signal of all pixels outside of the cell and within a $20 \mu \mathrm{m}$ region from the cell center.

To determine the response of a cell to each stimulus trial, the trace of a cell during the stimulation period was normalized to the baseline value averaged over the $0.5 \mathrm{~s}$ preceding stimulus presentation. The response of the cell to a given orientation, $\theta_{i}$, was defined as the average response across the eight repeats of each condition: $F\left(\theta_{i}\right)$. An estimate of the spontaneous calcium fluctuation of the cell was determined using the trace of the cell during the blank condition. At each spatiotemporal frequency, the responsiveness of a cell was determined using a one-way ANOVA [ $p<0.05 / n$ spatiotemporal stimulus conditions, with $n=20$ (5 $\mathrm{SF} * 4$ temporal frequency (TF)) stimulus conditions or $p<0.0025$ ] across stimulus trials against the blank condition. Only responses that passed the ANOVA test for at least one stimulus condition were considered significantly visually responsive. For the dataset concerning eyespecific responses (see eye specificity below), only visual responses that passed the significance threshold were included. For the dataset considering ocular dominance [see the Ocular dominance index (ODI) subsection below], responses were included regardless of meeting this criterion, as long as the cell met the $p<0.0025$ criteria for at least one of the spatiotemporal frequency conditions for one of the eye viewing conditions.

Orientation and direction selectivity. Orientation selectivity for a cell was determined using a method derived from the circular variance of the response of the cell $[F(\theta)$; Niell and Stryker, 2008; Kerlin et al., 2010; Hoy and Niell, 2015]. Since the circular variance-based method is sensitive to the sign of $F$ and because $F$ fluctuates above and below 0 at baseline $[\mathrm{SD}= \pm 0.032 \%$ response amplitude $(\mathrm{dF} / \mathrm{F})]$, we added an offset to $F$ for each cell, which set the minimum average response to $\left.0: F\left(\theta_{i}\right)\right)=F\left(\theta_{i}\right)-\min \left(\mathrm{F} \theta_{i}\right)$. Following this correction, the orientation selectivity index (OSI) was calculated as follows:

$$
\begin{gathered}
\text { OSI }=\left(\sqrt{\left(\sum_{i}\left(F\left(\theta_{i}\right) * \sin \left(2 \theta_{i}\right)\right)\right)^{2}+\left(\sum_{i}\left(F\left(\theta_{i}\right) * \cos \left(2 \theta_{i}\right)\right)\right)^{2}} /\right. \\
\left.\sum_{i} F\left(\theta_{i}\right)\right) .
\end{gathered}
$$

The direction selectivity index (DSI) was calculated as follows:

$$
\begin{gathered}
\text { DSI }=\left(\sqrt{\left(\sum_{i}\left(F\left(\theta_{i}\right) * \sin \left(\theta_{i}\right)\right)\right)^{2}+\left(\sum_{i}\left(F\left(\theta_{i}\right) * \cos \left(\theta_{i}\right)\right)\right)^{2}} /\right. \\
\left.\sum_{i} F\left(\theta_{i}\right)\right) .
\end{gathered}
$$

Preferred speed. To determine the preferred speed, responses across all spatial and temporal frequencies were fit with a two-dimensional elliptical Gaussian (Priebe et al., 2006; Andermann et al., 2011):

$$
\begin{gathered}
R(s f, t f)=A \exp \left(\frac{-\left(\log _{2} s f-\log _{2} s f_{o}\right)^{2}}{2\left(\sigma_{s f}\right)^{2}}\right) \\
\exp \left(\frac{-\left(\log _{2} t f-\log _{2} t f_{p}(s f)\right)^{2}}{2\left(\sigma_{t f}\right)^{2}}\right),
\end{gathered}
$$

where $A$ is the maximum responses of the neuron, $s f_{o}$ and $t f_{o}$ are the preferred spatial and temporal frequency, and $\sigma_{s f}$ and $\sigma_{t f}$ are the tuning widths for spatial and temporal frequency. From this fit, we are able to obtain the dependence of temporal frequency preference on spatial frequency by calculating the speed-tuning index $\xi$, such that $\log _{2} t f_{p}(s f)=\xi\left(\log _{2} s f-\log _{2} s f_{o}\right)+\log _{2} t f_{o}$. A neuron with a speed-tuning index of $\xi \approx 1$ is a speed-tuned cell, while $\xi \approx 0$ is not speed tuned and $\xi \approx-1$ is antituned. To measure goodness of fit, we used two approaches. First, the fitted data must be well correlated with the raw data (fit correlation, >0.5). The confidence intervals for preferred spatial and temporal frequency must not exceed 2 octaves. Overall, 4390 cells were considered responsive and well fit of the 12,852 ROIs identified. For speed tuning index analysis, we applied the additional criteria that the confidence intervals for the speed-tuning index must not exceed 1 (see Fig. 9). For the speed-tuning index analysis, imposing this criterion excluded 4103 of 4390 cells, or $93 \%$ of the cells.

Eye specificity. Eye specificity was calculated as $(C-I) /(C+I)$, where $C$ is the maximum response of the neuron $(A)$ for the contralateral eye and $I$ is the maximum response of the neuron for the ipsilateral eye. Thus, $A$ was taken at the optimal spatiotemporal frequency of the cell for 
A

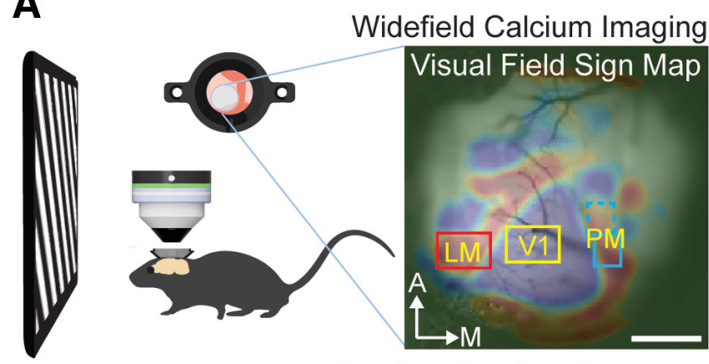

Two-photon GCaMP6s Imaging

Visual Stimuli

4 TFs: $1-8 \mathrm{~Hz}$, log-spaced

$5 \mathrm{SFs}: 0.03-0.48 \mathrm{c} / \mathrm{d}$, log-spaced

4 directions: $0,90,180,270^{\circ}$

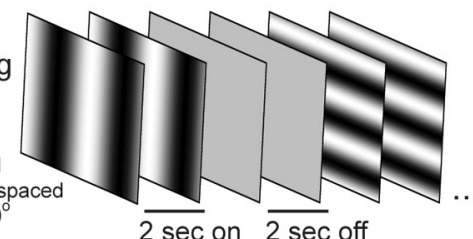

B
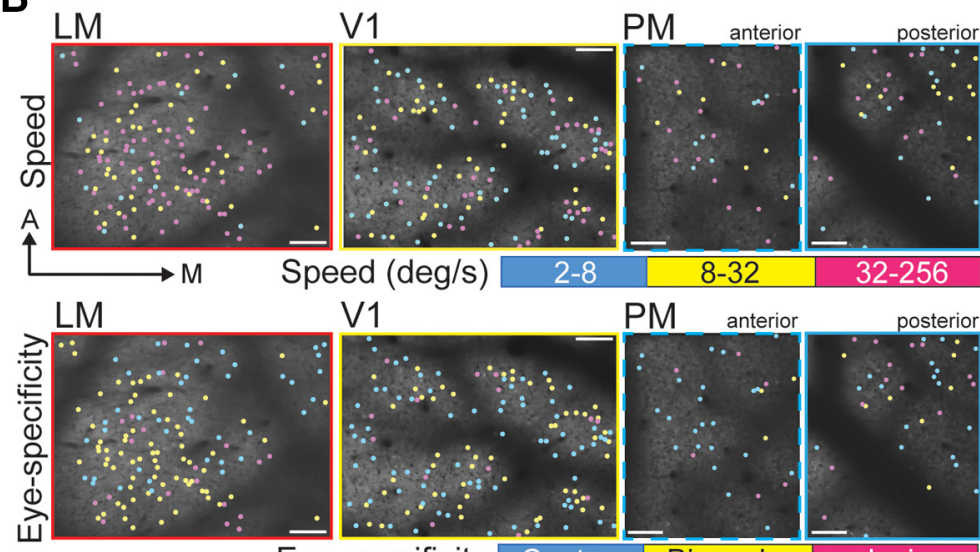

Eye-specificity Contra Binocular Ipsi V1 PM anterior
C

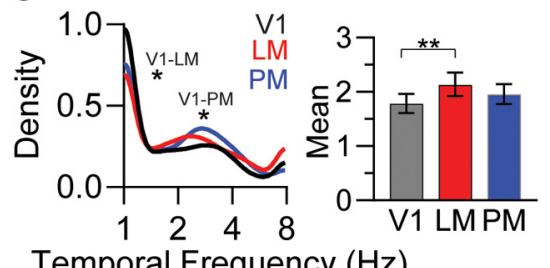

D

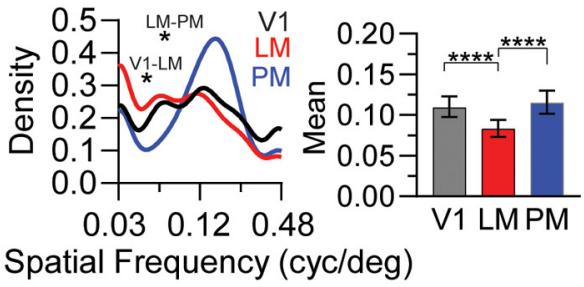

$\mathbf{F}$
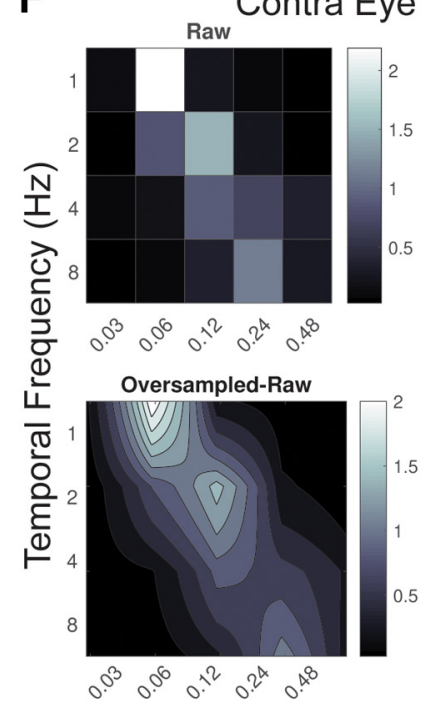

Viewing $_{\text {Fit }}$

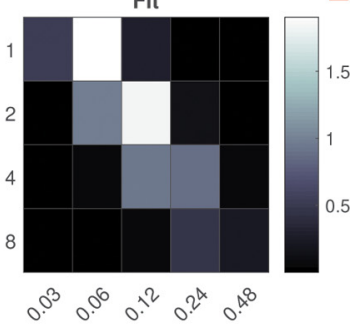

Oversampled-Fit

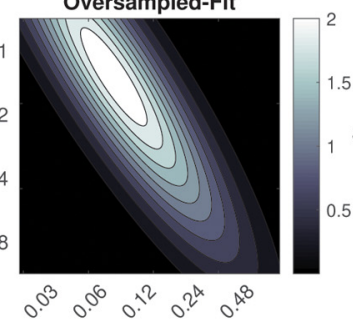

E

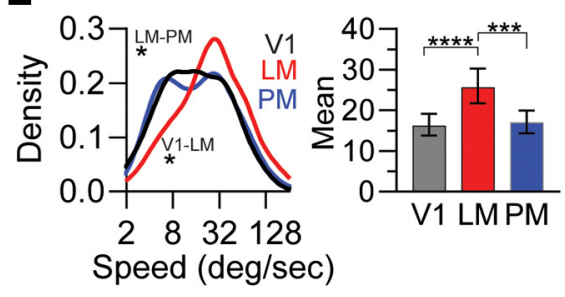

Example LM Cell Contra fit: 5 SFp:0.082 cyc/deg TFp: $1.61 \mathrm{~Hz}$

Speed:19.6 deg/sec Epsilon:1

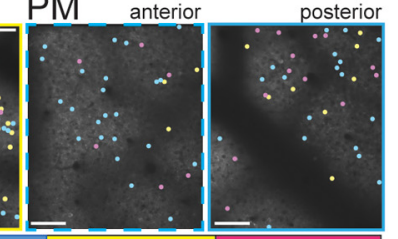

Ipsi Eye Viewing

Spatial Frequency (cycles/degree)
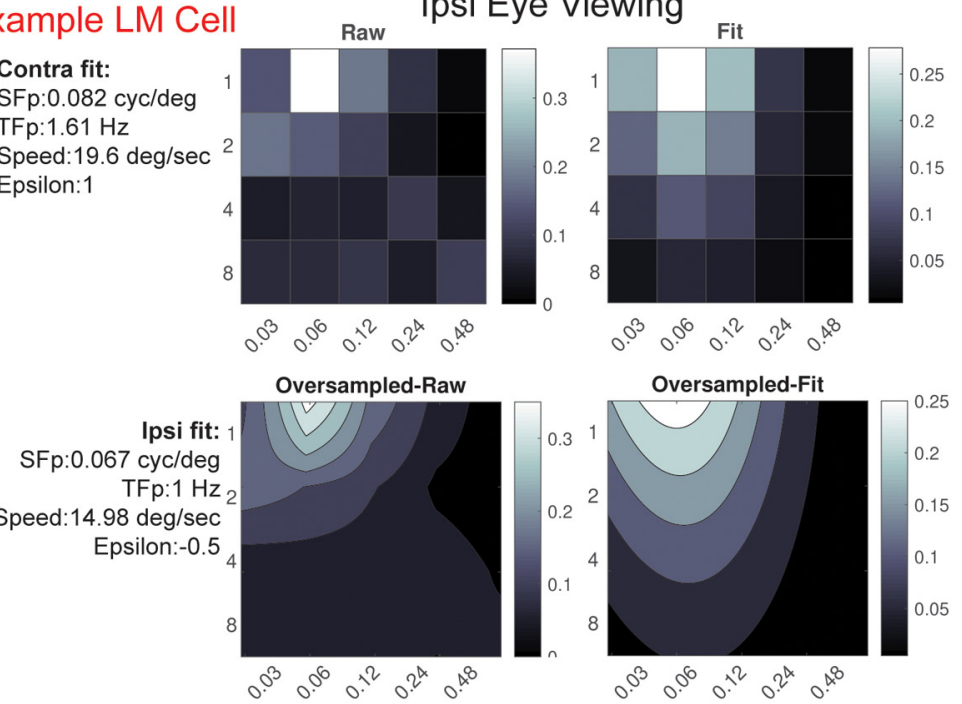

Figure 1. Probing spatiotemporal and eye-specific response properties of V1, LM, and PM. $A$, Experimental setup. Top right, Wide-field visual field sign map was used to delineate visual areas. Scale bar, $1 \mathrm{~mm}$. Top left, Two-photon calcium imaging experiments were centered in V1, LM, or PM to record neural activity while mice viewed drifting sinusoidal gratings of various spatial and temporal frequencies (speeds). The stimulus was shown to either the contralateral or ipsilateral eye to generate eye-specific speed-tuning curves. $\boldsymbol{B}$, Top, Maps of speed preferences for LM, V1, and PM from the same animal. Bottom, Maps of eye specificity for the same fields as above. Scale bars, $100 \mu \mathrm{m}$. $\mathbf{C}-\boldsymbol{E}$, Left, Density plots of preference distributions for cells recorded in V1, LM, and PM in control mice (C, temporal frequency; D, spatial frequency; E, speed). Right, Bootstrapped means. $F$, Spatiotemporal response matrices for an example binocular cell in LM. Raw data (left, top) were oversampled (left, bottom) and fit with a two-dimensional Gaussian (right, top and bottom), used to find preferred spatial and temporal frequencies for both contralateral eye viewing (left) and ipsilateral eye viewing (right). Error bars in $\boldsymbol{C}-\boldsymbol{E}$ represent confidence intervals. For density plots in $\boldsymbol{C}-\boldsymbol{E}$, $*$ represents statistical significance with multiple-comparisons correction $(p<0.05 / 3)$. For bootstrapped mean plots, ${ }^{*} p<0.05 ;{ }^{* *} p<0.01 ;{ }^{* * *} p<0.001$; ${ }^{* * * *} p<0.0001$.

each eye viewing condition. We classified a cell as contralateral eye dominated if the cell was only significantly responsive during contralateral eye viewing conditions. These cells were assigned an eye specificity of 1 . A cell that was only responsive during the ipsilateral eye viewing condition was considered ipsilateral eye dominated and assigned an eye specificity of -1 . When comparing cellular distributions for different spatial, temporal, and speed preferences, we used the response of the dominant eye if the cell was binocular. The dominant eye was determined based on which eye viewing condition led to a greater response amplitude.

Ocular dominance index. The ocular dominance index was calculated as $(C-I) /(C+I)$, where $C$ is the the maximum response of the neuron $(A)$ for the contralateral eye and $\mathrm{I}$ is the maximum response of the neuron for the ipsilateral eye. Thus, $A$ was taken at the optimal spatiotemporal frequency of the cell for each eye viewing condition. The response of the nondominant eye was used to calculate ODI, regardless 
Table 1. Visual properties in NoMD mice

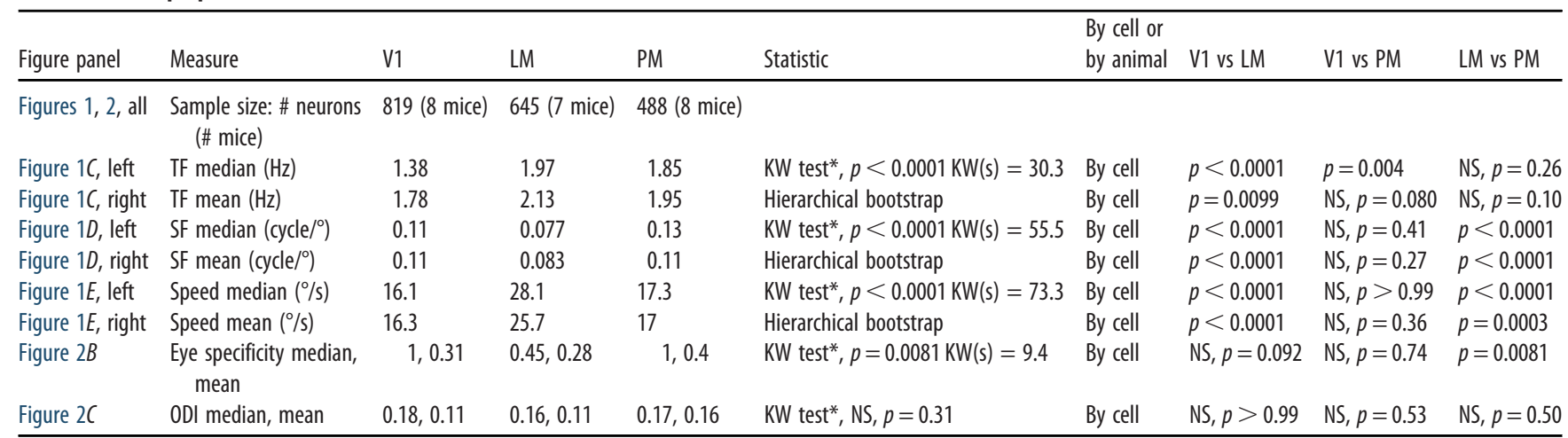

KW, Kruskal-Wallis test. TF, SF, TF/SF, eye specificity, and ODI preferences for cells that were visually responsive in each area in NoMD mice. KW(s) designates the statistic for the Kruskal-Wallis test. The larger the value of $s$ the larger the difference in the rank sums.

* Statistics with a multiple-comparisons correction.
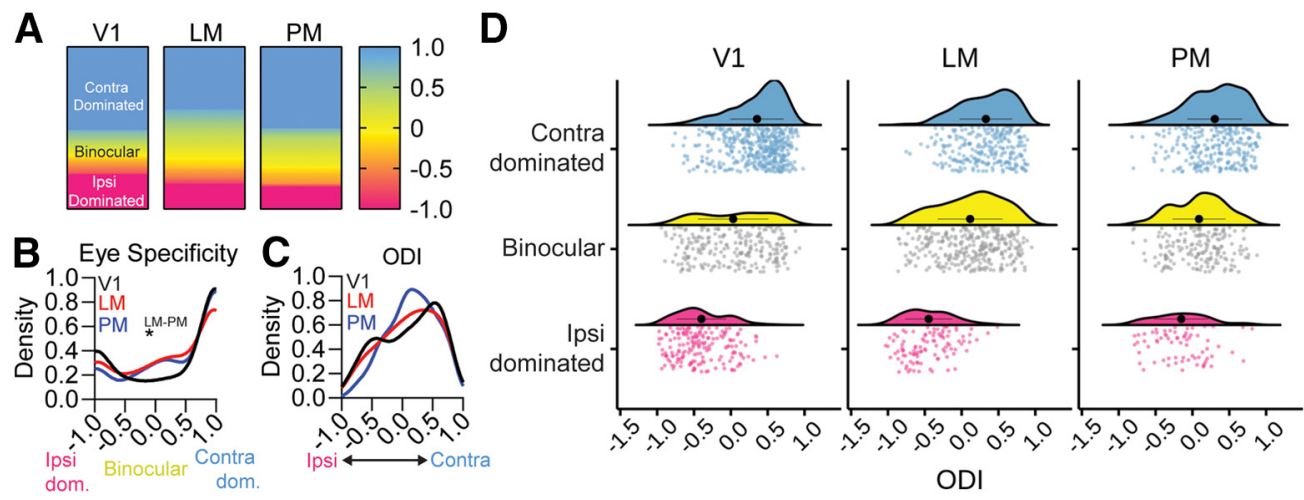

Figure 2. Comparison of eye specificity and ocular dominance in V1, LM, and PM. A, Relative proportions of cells categorized by eye specificity in V1, LM, and PM (see Materials and Methods for the definition of eye specificity). B, Density plot for eye-specific responses in V1, LM, and PM. C, Density plot for ocular dominance index (ODI) in V1, LM, and PM (see Materials and Methods for the computation of ODI). D, Rain cloud plots of ODI for eye-specific responses in V1 (left), LM (middle), and PM (right). Black filled circles represent the mean, and error bars indicate SD. For density plots * represents statistical significance with multiple-comparisons correction $(p<0.05 / 3)$.

of whether the responses were significantly driven during the viewing conditions of the nondominant eye.

\section{Experimental design and statistical analyses}

To compare temporal frequency preferences, spatial frequency preferences, speed preferences, eye specificity, ocular dominance indices, and speed-tuning indices among V1, LM, and PM in control (NoMD), CMD, and IMD mice, the Kruskal-Wallis test was used. Similarly, we used a Kruskal-Wallis test to compare intra-areal eye-specific speed preferences in NoMD, CMD, and IMD mice. A Bonferroni correction was applied to the Kruskal-Wallis test, such that $p<0.05 / 3$ or $p<0.0167$ was used as a threshold for significance. To demonstrate the robustness of the findings in the differences between distributions, we also performed a hierarchical bootstrap method on the means of the distributions (Saravanan et al., 2019). Following the methods outlined by Saravanan et al. (2019), we used the distribution of bootstrapped sample means of two groups of interest to compute probabilities ( $p$ values) that the data support the hypothesis that the two groups belong to the same distribution. This was done by plotting the bootstrapped sample mean distributions against one another in a $2 \mathrm{D}$ plot and calculating directly the proportion of sample means that were found on one side of the unity line.

To quantify the variable importance for DSI, OSI, dF/F, temporal frequency, spatial frequency, speed, and eye specificity for differentiating between visual areas, we used a regression random forest analysis (bagging method, random with replacement, 100 trees, XLSTAT). Additionally, the four most important variables were then used to quantify their relative variable importance on differentiating between different pairs of visual areas (XLSTAT).

To quantify functional segregation of HVAs and eye-specific responses, we first used a Box-Cox transformation (XLSTAT), optimized for all control data, to achieve distributions of spatial and temporal frequency that were more likely to be normally distributed. We then ran the Mahalanobis distance test with a Bonferroni correction for the number of comparisons (XLSTAT) on all cellular spatial and temporal frequency preferences for by-cell analysis, or on mean preferences obtained from each animal for by-animal analysis. For comparisons of HVA functional segregation, the interareal Mahalanobis distances for each animal in NoMD and CMD or IMD mice were then tested with a two-way ANOVA. The interareal distances for NoMD and CMD or IMD mice were summed and then compared with Welch's unpaired $t$ test to determine whether summed Mahalanobis distances were impaired with CMD or IMD. Statistical analyses were performed using Prism version 7.01 (GraphPad), XLSTAT, and MATLAB. The exact statistical tests and values for each figure are listed in the tables.

\section{Results}

To probe the functional response properties in mouse visual cortex, we used a transgenic line that expresses GCaMP6s under the control of the CaMK2 promoter (CaMK2-tTA; tetO-GCaMP6s; Wekselblatt et al., 2016). We used the visual field sign map generated by wide-field calcium imaging to delineate area boundaries. Next, we imaged visually evoked activity of excitatory 
A

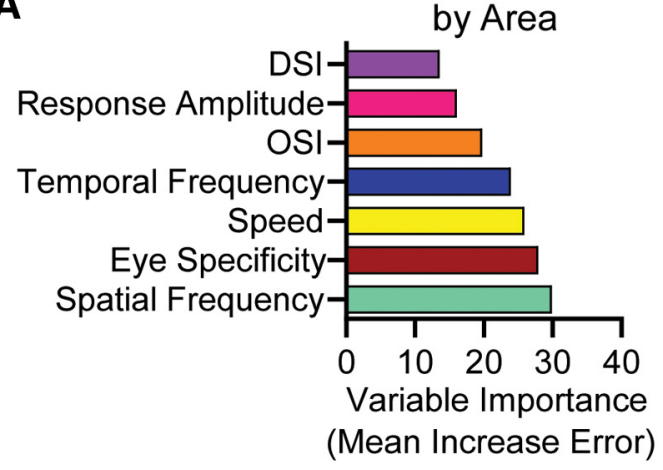

B

\section{Pair-wise Comparisons}

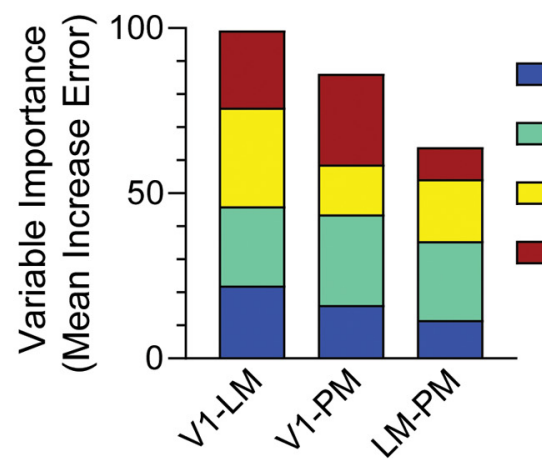

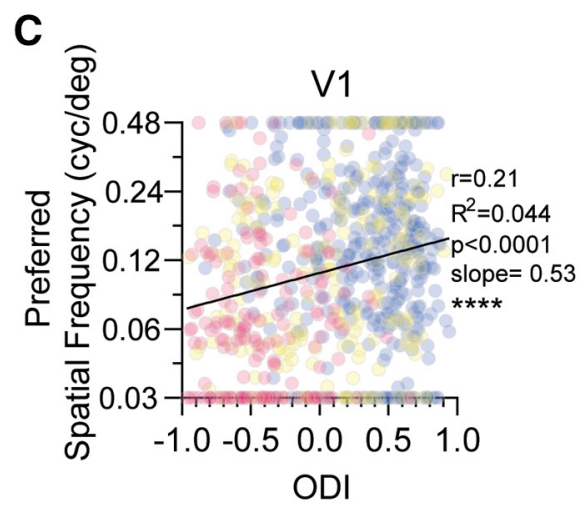
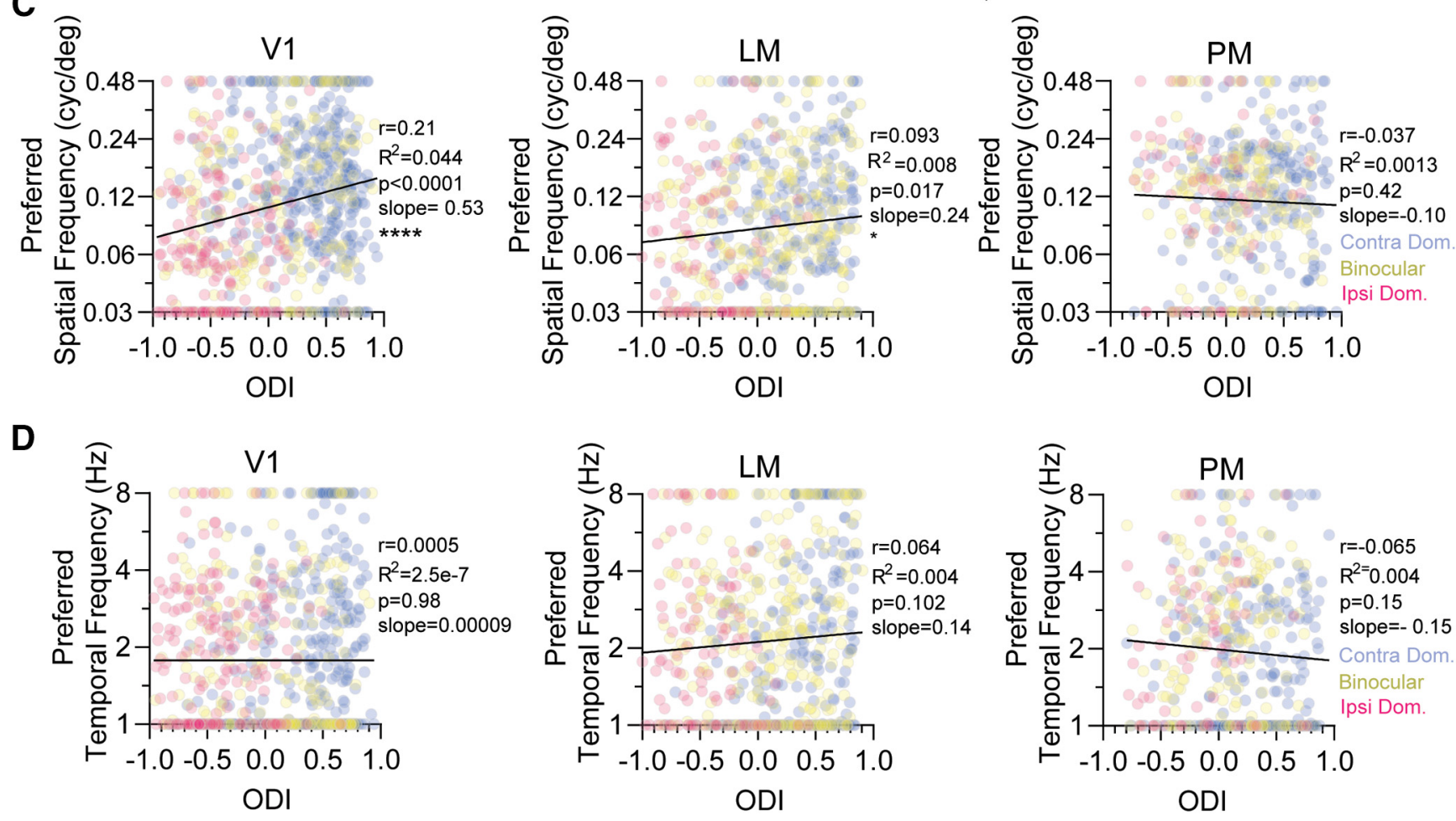

Figure 3. Spatiotemporal tuning and eye specificity are important features distinguishing visual areas. $A$, Results from a random forest classification performed on multiple parameters [direction selectivity (i.e., DSI), response amplitude, orientation selectivity (i.e., OSI), temporal frequency, speed, eye specificity, and spatial frequency] between areas V1, LM, and PM. $\boldsymbol{B}$, Pairwise interareal comparisons for the four most important variables. C, Relationship between preferred spatial frequency and ocular dominance index (ODI) of cells recorded within V1 (left), LM (middle), and PM (right) shown using scatter plots (linear regression overlaid in black). Data points are color coded by eye specificity (blue, contralateral dominated; yellow, binocular; pink, ipsilateral dominated). D, Relationship between preferred temporal frequency and ocular dominance index of cells recorded within V1 (left), LM (middle), and PM (right) shown using scatter plots (linear regression overlaid in black). For linear regression slopes ${ }^{*} p<0.05$; ${ }^{* *} p<0.01$; ${ }^{* * *} p<0.001$; ${ }^{* * * *} p<0.0001$.

neurons in L2/3 of adult mice across areas V1, LM, and PM (Fig. $1 A)$. By presenting drifting sinusoidal gratings of various temporal and spatial frequencies to each eye individually, we assessed eye-specific tuning for spatial frequency, temporal frequency, and speed (Fig. $1 B-F$ ). The spatiotemporal frequency, or speed, of these gratings was defined as the temporal frequency/spatial frequency. It has been shown that HVAs can be functionally segregated more robustly using speed preferences over spatial frequency preferences alone (Glickfeld et al., 2013). Response matrices for each eye viewing condition were fit with a twodimensional Gaussian (Priebe et al., 2006; Andermann et al., 2011; Glickfeld et al., 2013) to generate spatial and temporal frequency-tuning curves and estimate spatiotemporal preferences (preferred speed $=$ preferred temporal frequency/preferred spatial frequency; Fig. $1 F)$.

\section{Distinct response properties of $\mathrm{V} 1, \mathrm{LM}$, and $\mathrm{PM}$}

In adult mice, neurons in $\mathrm{V} 1, \mathrm{LM}$, and PM respond distinctly to spatiotemporal information (Fig. $1 C-E$ ). V1 is tuned to relatively slower temporal frequencies than LM and PM (Fig. 1C, Table 1). LM is tuned to relatively lower spatial frequencies than V1 and PM (Fig. 1D, Table 1). The combined differences in spatial and temporal frequency preferences result in unique spatiotemporal frequency preferences for V1, LM, and PM (Fig. 1E, Table 1). While all three areas contain neurons that respond to each or both eyes, neurons in the HVAs differ in their distribution of contralateral dominated, binocular, and ipsilateral dominated neurons (Fig. 2A,B, Table 1). Cells in LM, which is highly binocular (Fig. $2 A, B$ ), prefer relatively lower spatial frequencies and faster speeds (Fig. $1 D, E$ ). In contrast, area PM contains a high proportion of contralateral dominated cells with fewer ipsilateral 
dominated cells and is tuned to relatively higher spatial frequencies and slower speeds (Fig. 1D,E).

We have previously reported that contralateral dominated neurons are functionally distinct from binocular and ipsilateral dominated neurons in V1 (Salinas et al., 2017). In our previous articles (Salinas et al., 2017; Huh et al., 2020), and here, we applied a filter for visually responsive significance when calculating ocular dominance, which we refer to here as "eye specificity," whereby only visually driven responses that met our statistical criteria for responsivity are used in the calculation for eye specificity (Fig. 2A,B). Although other studies have used methods similar to ours (Jaepel et al., 2017; Jenks and Shepherd, 2020), we recognize that this is not a typical method for calculating the ODI, where the response of the nondominant eye is usually taken into consideration regardless of passing a statistical test. We therefore calculated ODI in a more conventional way as a means to relate our method to more traditional methods (Fig. 2C,D). Using this more liberal criterion of data inclusion, we did not find differences in ODI between areas (Table 1). In adult control mice, we find that contralateral and ipsilateral dominated neurons are highly biased to the contralateral or ipsilateral eye, respectively, but also contain a nondominant eye component that fails to pass visual significance in V1, LM, and PM (Fig. 2D, top, bottom). In contrast, binocular cells display intermediate ODI values between the contralateral and ipsilateral dominated ODI distributions in V1, LM, and PM (Fig. $2 D$, middle).

\section{Spatiotemporal tuning and eye specificity are important features distinguishing visual areas}

To take an unbiased approach to determining what features are important for distinguishing among V1, LM, and PM, we performed a random forest analysis on neural data for multiple parameters including response amplitude, OSI, DSI, spatial frequency, temporal frequency, speed, and eye specificity. Random forest analysis revealed that eye specificity, speed, spatial frequency, and temporal frequency are the most important features distinguishing among V1, LM, and PM in adult control mice (Fig. $3 A, B$ ). Since eye specificity was shown to be as important as temporal frequency (TF), spatial frequency (SF), and spatiotemporal frequency (TF/SF, or speed) tuning, we asked whether there was a relationship between ODI and TF, SF or speed in V1, LM and PM. In V1, spatial frequency is correlated with ODI (Fig. 3C, left) while temporal frequency is not (Fig. $3 D$, left). In LM, spatial frequency is correlated with ODI (Fig. 3C, middle) but temporal frequency is not (Fig. 3D, middle). Last, in PM, neither spatial frequency nor temporal frequency is correlated with ocular dominance index (Fig. $3 C$, right, $D$, right). Together, ODI is negatively correlated with preferred speed in V1 (see Fig. 5A, left), but not LM or PM (see Fig. 5A, middle, right).

Previously, we reported that contralateral dominated neurons in V1 were tuned to higher spatial frequencies than binocular or ipsilateral dominated neurons (Salinas et al., 2017). Here we report that ODI is negatively correlated with speed preferences in V1, but not $\mathrm{LM}$ or PM, in control mice. Given the unique speed preference profiles of V1, LM, and PM, we hypothesized that depriving mice of visual experience through one eye during the critical period for binocular vision may differentially alter the speed preferences of cells in V1, LM, and PM.

\section{Monocular deprivation alters the relationship between ODI and speed preferences in higher visual areas}

To determine whether the functional differentiation of HVAs depends on early binocular visual experience, we deprived mice of vision through the contralateral or ipsilateral eye for 2 weeks starting at P19 (CMD, IMD) and assessed eye-specific spatiotemporal tuning in adulthood. We used the visual field sign map to determine areal borders and found no differences in the sizes of visual areas in adulthood following visual deprivation (Fig. $4 A, B$, Table 2). We also found no evidence for a change in the percentage of visually responsive or well fit neurons in V1, LM, or PM following monocular deprivation (Fig. $4 C$, Table 2). These results suggest that some fundamental properties such as the size of the visual areas and visual responsiveness remain intact in adulthood following juvenile MD manipulations.

We asked whether the correlation between ODI and speed preferences is altered following CMD or IMD in V1, LM, and PM (Fig. 5). In V1, neither CMD nor IMD disrupts the negative correlation between ODI and speed preferences (Fig. 5B,C). In LM and PM, which normally have no correlation between ODI and speed preferences, IMD leads to a negative correlation between ODI and speed preferences (Fig. 5). This relationship is demonstrated quantitatively as the slope of the linear regression line, which is larger in LM and PM with both CMD and IMD compared with control (Fig. 5D). To summarize, in normally reared adult mice, ODI is negatively correlated with speed preferences in V1, but not LM and PM (Fig. 5D). Following deprivation, the general relationship between ODI and spatiotemporal tuning is preserved in V1 but is altered in LM or PM (Fig. 5D). Following deprivation, especially ipsilateral eye deprivation, LM and PM cells gain a negative correlation between ODI and speed preferences that resembles that of V1 (Fig. 5D). Thus, monocular deprivation of either eye produces a similar ODI and speed preference relationship for all three areas, resulting in areas that are functionally more similar.

\section{Monocular deprivation alters speed preferences in V1, LM, and PM differentially}

In V1, CMD and IMD result in a shift of the distribution for preferred spatial frequency toward lower spatial frequencies (Fig. 6B, Table 3) with no significant change in the distribution of preferred temporal frequency (Fig. 6A, Table 3). Overall, both CMD and IMD shift speed preferences toward faster speeds in V1 
Table 2. General properties of visual areas

\begin{tabular}{|c|c|c|c|c|c|c|}
\hline Figure panel & Measure / Statistic & Area & NoMD & CMD & IMD & By cell or by animal \\
\hline \multirow[t]{3}{*}{ Figure 4, all } & Sample size: \# mice & V1 & 8 mice & 6 mice & 6 mice & \\
\hline & & LM & 7 mice & 6 mice & 6 mice & \\
\hline & & PM & 8 mice & 6 mice & 6 mice & \\
\hline \multirow{2}{*}{ Figure $4 B$} & & LM & 0.93 & 1.04 & 1.02 & By animal \\
\hline & & PM & 0.51 & 0.58 & 0.59 & By animal \\
\hline Figure $4 B$ & One-way ANOVA on mean area size & V1 & NS, $p=0.19$ & & & By animal \\
\hline \multirow[t]{3}{*}{ Figure $4 C$, left } & Mean \% responsive & V1 & 54.3 & 51.4 & 58.6 & By animal \\
\hline & & LM & 57.6 & 61.3 & 65.9 & By animal \\
\hline & & PM & 39.8 & 41.7 & 36.5 & By animal \\
\hline Figure $4 C$, left & Two-way ANOVA on mean \% resp & Area $p=0.0003$; deprivation $p=0.87 ;$ area $\times$ deprivation $p=0.78$ & & & & By animal \\
\hline \multirow[t]{2}{*}{ Figure $4 C$, right } & Mean $\%$ well fit & V1 & 34.4 & 32.3 & 40 & By animal \\
\hline & & LM & 38.7 & 39.8 & 43.5 & By animal \\
\hline
\end{tabular}

Size of visual area, percentage of cells that were visually responsive, and percentage of cells that were considered well fit by the two-dimensional Gaussian and included in our analyses.

(Fig. 6C, Table 3). Although monocular deprivation did not significantly shift the individual distributions of preferred temporal frequency (Fig. 6D,G, Table 3) or spatial frequency (Fig. 6E, $H$, Table 3) in LM or PM, CMD results in a shift of speed preferences toward slower speeds in LM (Fig. 6F, Table 3) while IMD results in a shift of speed preferences toward faster speeds in PM (Fig. 6I, Table 3). Thus, contralateral and ipsilateral eye monocular deprivation results in differential shifts in spatiotemporal tuning in V1, LM, and PM (Fig. 6J-L).

\section{Monocular deprivation disrupts areal differentiation}

We next asked how monocular deprivation impacts the interareal differentiation of spatiotemporal tuning of V1, LM, and PM. Compared with control mice, where there are distinct spatiotemporal tuning differences among V1, LM, and PM (Fig. 1C-E), we find that interareal differences are generally weakened in CMD and IMD mice (Fig. 7A-F). Differences in speed preferences between areas were eliminated in CMD mice and largely abolished in IMD mice (except for the difference between V1 and LM in IMD; Fig. 7C,F, Tables 1, 4, 5). These effects are largely driven by the diminished differences between areas in terms of spatial frequency tuning (Figs. $1 D, 7 B, E$, Tables 1, 4, 5), whereas some of the temporal frequency tuning differences between areas are preserved (Figs. $1 C, 7 A, D$, Tables $1,4,5$ ). Overall, these findings indicate that there is disrupted interareal differentiation for spatiotemporal tuning following CMD and IMD (compare Figs. $7 C, F, 1 E)$.

To explicitly test whether interareal differences in spatiotemporal tuning are impacted by juvenile monocular deprivation, we used the Mahalanobis distance metric (Murakami et al., 2017). In controls, the spatiotemporal preferences of each area fall within distinct clusters (Fig. $7 G, H$, Table 6). Both CMD and IMD appeared to reorganize the functional clustering of visual areas (Fig. 7G,H, Table 6). To test the effect of MD on functional clustering, we compared the interareal Mahalanobis distances by animal. CMD had a significant effect on the interareal Mahalanobis distances (Fig. 7I, top, Table 6). Most notably, the Mahalanobis distance between $\mathrm{V} 1$ and LM in the spatiotemporal domain was greatly reduced (Fig. 7I, top, Table 6; LM vs V1, $p=0.0091$ ). By animal analysis demonstrates that CMD diminishes the summed interareal Mahalanobis distances, resulting in reduced functional differentiation between areas (Fig. 7I, bottom, Table 6). IMD also had a significant effect on the interareal distances between V1 and LM (Fig. 7J, top, Table 6), but overall the effects on the summed interareal distances were not significant (Fig. 7J, bottom, Table 6). Thus, both CMD and IMD reduced interareal differences in spatiotemporal tuning.

\section{Monocular deprivation alters eye-specific speed preferences within visual areas}

We previously reported distinct eye-specific functional tuning for spatial frequency in V1 neurons (Salinas et al., 2017). To address whether eye-specific neural responses are functionally differentiated in higher visual areas, we grouped neural responses into three eye-specific categories [Fig. 8: contralateral dominated (C), binocular (B), ipsilateral dominated (I) neurons] and tested whether their distributions for preferred speed were significantly different in control mice (Fig. $8 A-C$ ). In V1 and LM, neurons dominated by the contralateral eye inputs prefer the slowest speeds (Fig. 8A,B, Table 7), whereas in PM all eye-specific neural responses were generally tuned to relatively slow/intermediate speeds (Fig. 8C, Table 7). In V1, neurons linked by eye specificity are tuned to distinct spatiotemporal information, with binocular neurons preferring intermediate speeds and ipsilateral-dominated neurons preferring the fastest speeds (Fig. 8A). Ipsilateral dominated neurons of LM prefer significantly faster speeds than their contralateral dominated counterparts (Fig. 8B). In contrast, in PM, neurons linked by eye specificity are less distinct in their speed preferences compared with the other areas (Fig. 8C).

Neither CMD nor IMD led to a total elimination of the difference in eye-specific speed preferences in V1 since contralateral dominated cells are tuned to slower speeds compared with binocular and ipsilateral dominated cells in CMD and IMD (Fig. $8 D, G$, Tables 8,9$)$. In LM, many of the differences in eye-specific speed tuning also remain following CMD and IMD (Fig. $8 B, E, H$, Tables 8, 9). In PM, eye-specific speed-tuning differences are not significant in controls but become more pronounced following IMD, but not CMD (Fig. 8C,F,I, Tables 8, 9). Overall, contralateral and ipsilateral eye monocular deprivation results in differential shifts in spatiotemporal tuning of eye-specific responses in V1, LM, and PM (Fig. 8J-L).

To quantify the effect of MD on the functional differentiation of eye-specific responses in V1, LM, and PM, we 
A
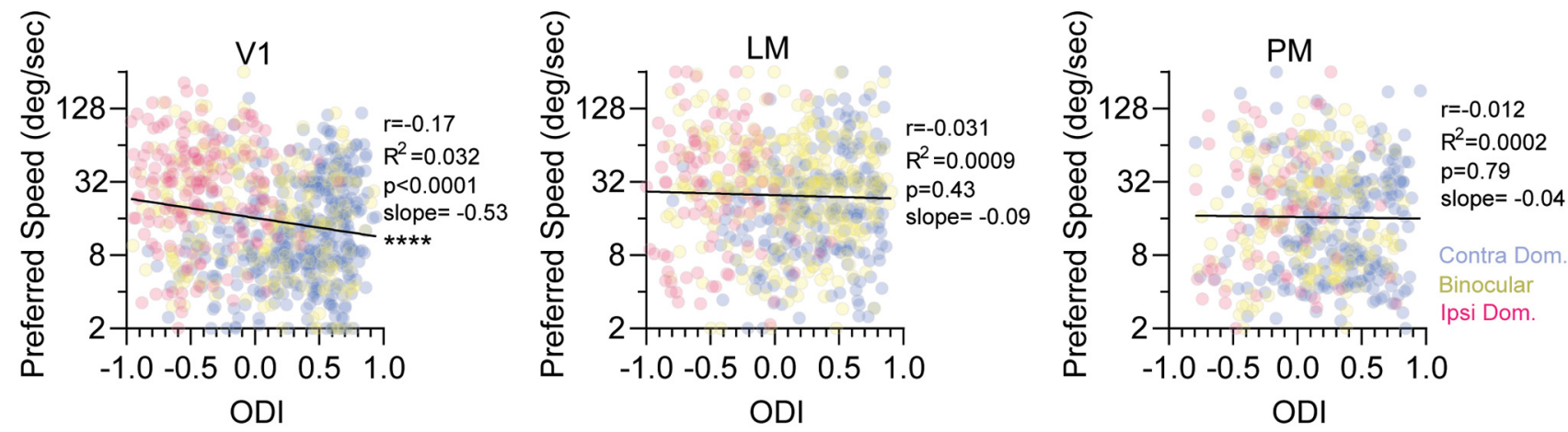

B
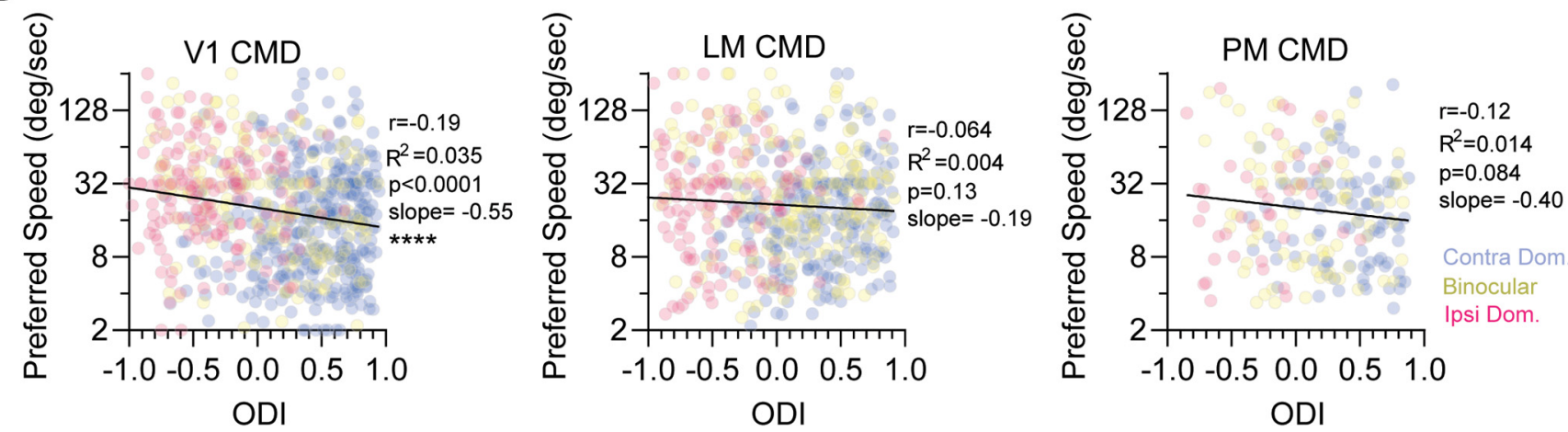

C
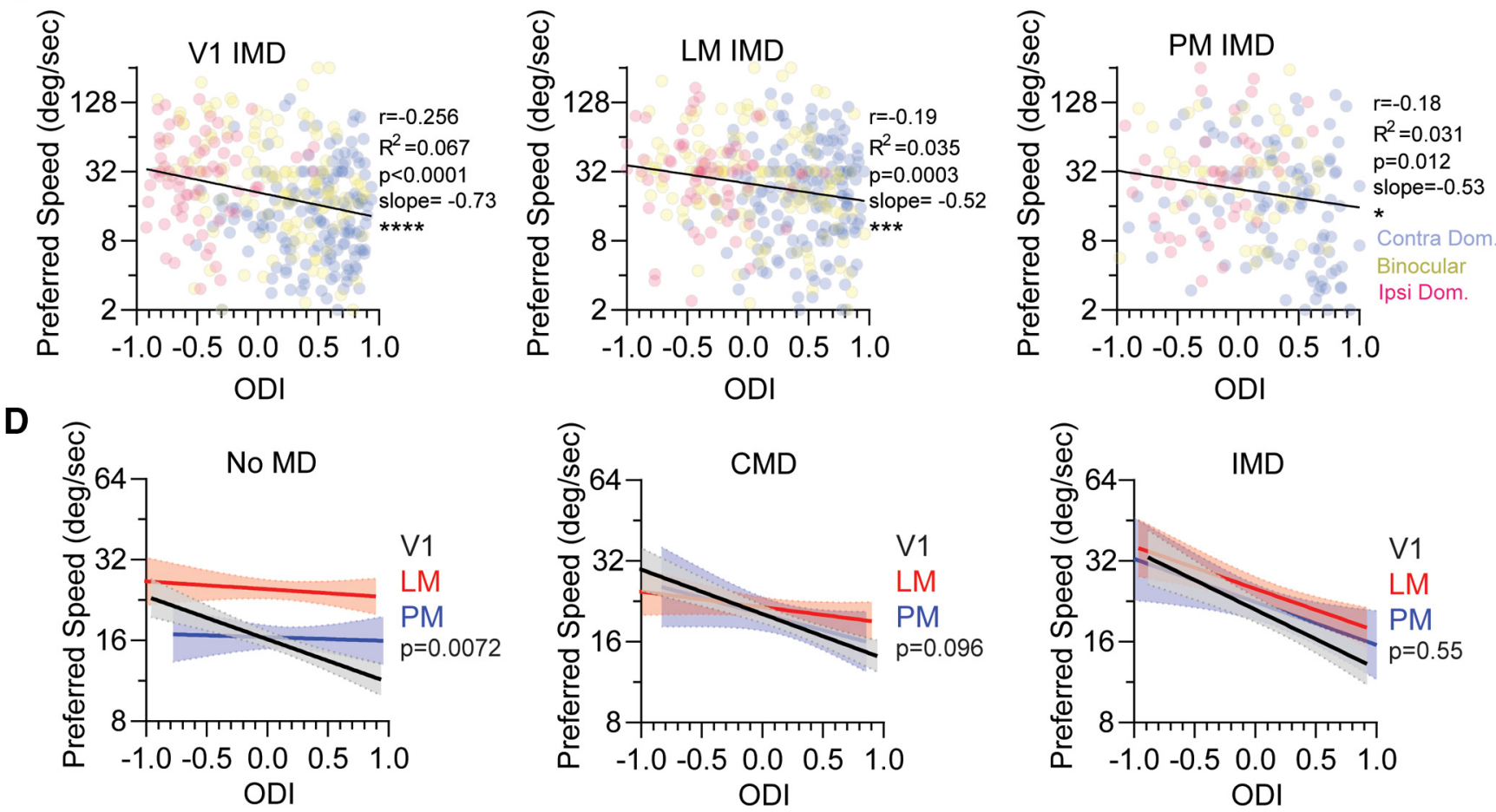

Figure 5. Effects of monocular deprivation on the relationship between ocular dominance and spatiotemporal tuning. $A$, Relationship between preferred speed and ocular dominance index (ODI) of cells recorded within V1 (left), LM (middle), and PM (right) of control mice shown using scatter plots (linear regression overlaid in black). Data points are color coded by eye specificity (blue, contralateral dominated; yellow, binocular; pink, ipsilateral dominated). $\boldsymbol{B}$, Relationship between preferred speed and ocular dominance index of cells recorded within V1 (left), LM (middle), and PM (right) of CMD mice shown using scatter plots (linear regression overlaid in black). C, Relationship between preferred speed and ocular dominance index of cells recorded within V1 (left), LM (middle), and PM (right) of IMD mice shown using scatter plots (linear regression overlaid in black). $\boldsymbol{D}$, Linear regressions with shaded SE describing the relationship between preferred speed and ODI overlaid for each area (V1, black; LM, red; PM, blue) in NoMD (right), CMD (middle), and IMD (right) mice. For linear regression slopes ${ }^{*} p<0.05$; ${ }^{* *} p<0.01$; ${ }^{* * *} p<0.001 ;{ }^{* * *} p<0.0001$. 
Table 3. Effect of CMD and IMD on visual properties

\begin{tabular}{|c|c|c|c|c|c|c|c|c|c|}
\hline Figure panel & Measure & Area & NoMD & CMD & IMD & Statistic & $\begin{array}{l}\text { By cell or by } \\
\text { animal }\end{array}$ & NoMD vs CMD & NoMD vs IMD \\
\hline \multirow[t]{3}{*}{ Figure 6, all } & \multirow[t]{3}{*}{ Sample size: \# neurons (\# mice) } & V1 & 819 (8 mice) & 726 (6 mice) & 357 (4 mice) & & & & \\
\hline & & $\mathrm{LM}$ & 645 (7 mice) & 567 (4 mice) & 370 (4 mice) & & & & \\
\hline & & PM & 488 (8 mice) & 216 (4 mice) & 202 (4 mice) & & & & \\
\hline \multirow[t]{3}{*}{ Figure $6 A$, left } & \multirow[t]{3}{*}{ TF median (Hz) } & V1 & 1.38 & 1.4 & 1.67 & KW test ${ }^{*}, \mathrm{NS}, p=0.22$ & By cell & NS, $p>0.99$ & $\mathrm{NS}, p=0.17$ \\
\hline & & $\mathrm{LM}$ & 1.97 & 1.91 & 1.88 & KW test ${ }^{*}, \mathrm{NS}, p=0.44$ & By cell & $\mathrm{NS}, p=0.41$ & NS, $p>0.99$ \\
\hline & & PM & 1.85 & 2.21 & 2.21 & KW test ${ }^{*}, \mathrm{NS}, p=0.04$ & By cell & $\mathrm{NS}, p=0.21$ & NS, $p=0.037$ \\
\hline \multirow[t]{3}{*}{ Figure $6 A$, right } & \multirow[t]{3}{*}{ TF mean $(\mathrm{Hz})$} & V1 & 1.78 & 1.83 & 1.88 & Hierarchical bootstrap & By cell & $\mathrm{NS}, p=0.52$ & $\mathrm{NS}, p=0.21$ \\
\hline & & LM & 2.13 & 2.05 & 2.08 & Hierarchical bootstrap & By cell & $\mathrm{NS}, p=0.28$ & NS, $p=0.38$ \\
\hline & & PM & 1.95 & 2.17 & 2.25 & Hierarchical bootstrap & By cell & NS, $p=0.053$ & NS, $p=0.11$ \\
\hline \multirow[t]{3}{*}{ Figure $6 B$, left } & \multirow[t]{3}{*}{ SF median $\left(\right.$ cycle $\left./{ }^{\circ}\right)$} & V1 & 0.11 & 0.088 & 0.091 & $\mathrm{KW}$ test ${ }^{*}, p=0.0002, \mathrm{KW}(\mathrm{s})=17.0$ & By cell & $p=0.0002$ & $p=0.009$ \\
\hline & & $\mathrm{LM}$ & 0.077 & 0.096 & 0.077 & KW test ${ }^{*}, \mathrm{NS}, p=0.037$ & By cell & NS, $p=0.027$ & NS, $p>0.99$ \\
\hline & & PM & 0.13 & 0.12 & 0.106 & KW test ${ }^{*}, \mathrm{NS}, p=0.14$ & By cell & NS, $p=0.65$ & NS, $p=0.10$ \\
\hline \multirow[t]{3}{*}{ Figure $6 B$, right } & \multirow[t]{3}{*}{ SF mean $\left(\right.$ cycle $\left./^{\circ}\right)$} & V1 & 0.109 & 0.093 & 0.094 & Hierarchical bootstrap & By cell & $p=0.03$ & $p=0.03$ \\
\hline & & LM & 0.083 & 0.093 & 0.086 & Hierarchical bootstrap & By cell & NS, $p=0.10$ & NS, $p=0.35$ \\
\hline & & PM & 0.12 & 0.11 & 0.1 & Hierarchical bootstrap & By cell & NS, $p=0.29$ & $\mathrm{NS}, p=0.23$ \\
\hline \multirow[t]{3}{*}{ Figure $6 C$, left } & \multirow[t]{3}{*}{ Speed median (deg/sec) } & V1 & 16.1 & 19.4 & 20.4 & KW test ${ }^{*}, p=0.0003, \mathrm{KW}(\mathrm{s})=16.3$ & By cell & $p=0.0008$ & $p=0.0035$ \\
\hline & & $\mathrm{LM}$ & 28.1 & 22.2 & 28.3 & KW test, $p=0.015, \mathrm{KW}(\mathrm{s})=8.40$ & By cell & $p=0.010$ & NS, $p>0.99$ \\
\hline & & PM & 17.3 & 21.1 & 25.3 & KW test ${ }^{*}, p=0.006, K W(s)=10.4$ & By cell & NS, $p=0.14$ & $p=0.0042$ \\
\hline \multirow[t]{3}{*}{ Figure $6 C$, right } & \multirow[t]{3}{*}{ Speed mean (deg/sec) } & V1 & 16.3 & 19.8 & 20.1 & Hierarchical bootstrap & By cell & $p=0.028$ & $p=0.04$ \\
\hline & & LM & 25.6 & 22 & 24.3 & Hierarchical bootstrap & By cell & NS, $p=0.11$ & NS, $p=0.35$ \\
\hline & & PM & 17 & 20 & 22.3 & Hierarchical bootstrap & By cell & NS, $p=0.086$ & $p=0.014$ \\
\hline
\end{tabular}

KW, Kruskal-Wallis test. TF, SF, and TF/SF preferences of cells that were visually responsive in each area in NoMD, CMD, or IMD mice.

*Statistics with a multiple-comparisons correction.

used the Mahalanobis distance metric. In V1, binocular spatiotemporal preferences are no longer distinct from the ipsilateral-dominated preferences following both CMD and IMD (Fig. 8M, first column: No MD, summed Mahalanobis distance $=1.42$; CMD, summed Mahalanobis distance $=0.68$; IMD, summed Mahalanobis distance $=1.12$; Fig. $8 N$, top). In LM, eyespecific spatiotemporal preferences are less functionally distinct following CMD and are more distinct following IMD [Fig. $8 \mathrm{M}$, middle column: No MD, summed Mahalanobis distance $=0.31$ (Table 10); CMD summed Mahalanobis distance $=0.23$ (Table 10); IMD, Summed Mahalanobis distance $=0.42$ (Table 10); Fig. $8 N$, middle]. In PM, the eye-specific spatiotemporal preferences are more functionally distinct following both CMD and IMD, but especially following IMD [Fig. 8M, left column: No $\mathrm{MD}$, summed Mahalanobis distance $=0.26$ (Table 10); CMD, summed Mahalanobis distance $=0.55$ (Table 10); IMD, summed Mahalanobis distance $=0.77$ (Table 10); Fig. $8 N$, bottom]. These results, together with those presented in Figure 5, indicate that MD has distinct effects on functional differentiation of eye-specific signals in different visual areas, such that the eye-specific signals are preserved but less distinct in V1 following CMD and IMD, while eye-specific signals become more distinct in LM and PM following IMD.

\section{Monocular deprivation disrupts the relationship between ocular dominance and degree of speed tuning in V1}

Mouse visual areas are not only selective for unique spatiotemporal information, but also have varying degrees of speed tuning or the dependence of temporal frequency preference on spatial frequency (Andermann et al., 2011; Roth et al., 2012; Fig. 1F, left, speed-tuned example, $F$, right, not speed-tuned example). We assessed the interareal differences of the degree of speed tuning in V1, LM, and PM and asked whether MD alters the degree of speed tuning (Fig. 9A). Speed-tuning indices indicate the degree of speed tuning of a cell, as follows: speed tuned $(\xi \approx 1)$, not speed tuned $(\xi \approx 0)$, or anti-tuned $(\xi \approx-1)$. We found no difference in the distributions for speed-tuning indices between areas, although we did notice that HVAs have a higher proportion of speed-tuned $(\xi \approx 1)$ cells than $\mathrm{V} 1$, similar to what has been previously reported in mice (Fig. 9A, left, Table 11; Andermann et al., 2011). CMD exaggerated the difference in the proportion of speed-tuned cells between V1 and PM, while IMD did not (Fig. 9A, middle, right, Table 11). Overall, MD did not disrupt the expression of speed-tuned cells in LM or PM.

Since the degree of speed tuning has been demonstrated to negatively correlate with speed preferences (Andermann et al., 2011), and, because contralateral-dominated responses are preferentially tuned to slower speeds in V1 (Figs. 5A, 8A), we tested whether there is a relationship between speed-tuning index and ODI in control and MD mice. In control mice, ODI is positively correlated with the speed-tuning index in all three visual areas (Fig. 9B). Linear regression analysis revealed that V1, LM, and PM have similar slopes but different intercepts, because of the overall higher degree of speed tuning in LM and PM (Fig. 9C, left: V1, $y$-intercept $=0.23$; LM, $y$-intercept $=0.43$; PM, $y$-intercept $=0.41, p=0.035)$. Interestingly, CMD and IMD disrupt the relationship between speed-tuning index and ODI, flattening the linear regression slope in V1 (Fig. 9C, middle, right). However, CMD did not disrupt the speed-tuning index-ODI relationship in LM or PM (Fig. 9C, middle: LM, $r=0.42 p=0.0094$; PM, $r=0.56, p=0.0031)$. In IMD mice, there is no significant relationship between speed-tuning index and ODI in any of the areas (Fig. 9C, right: V1, $p=0.93 ; \mathrm{LM}, p=0.25 ; \mathrm{PM}, p=0.52$; intercept, $p=0.36)$. Our results suggest that MD during the critical period disrupts the relationship between ODI and speed tuning index in V1 without causing significant impairments in the degree of speed tuning in higher visual areas.

\section{Discussion}

In this study, we find three pronounced effects of MD during the critical period on the functional differentiation of adult visual cortical areas. First, the functional differentiation of three visual areas, V1, LM, and PM, collapses following juvenile deprivation, 


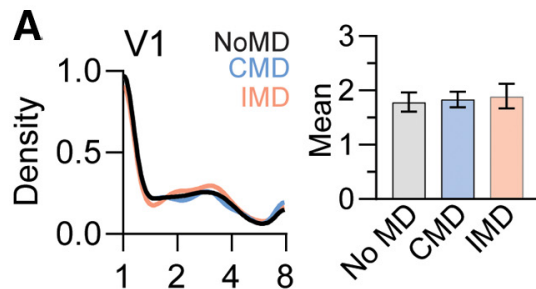

Temporal Frequency $(\mathrm{Hz})$

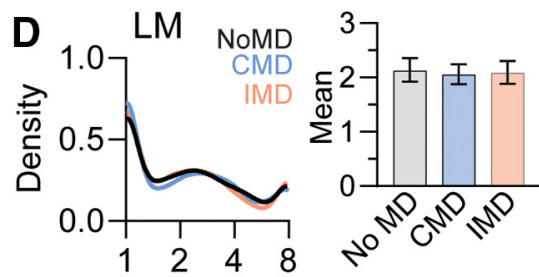

Temporal Frequency $(\mathrm{Hz})$
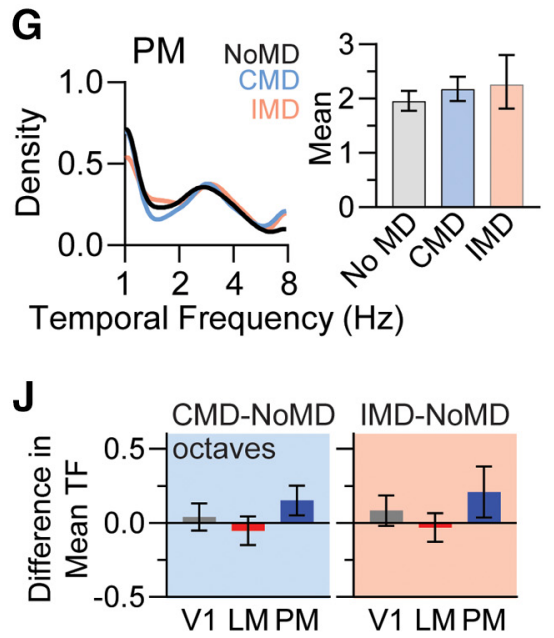
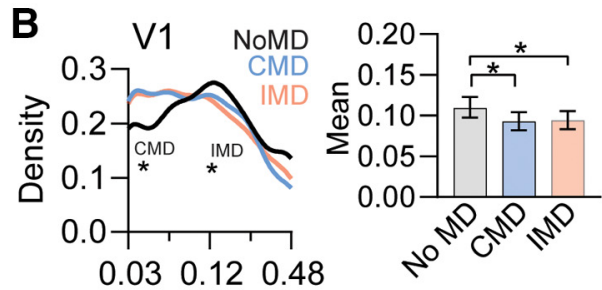

Spatial Frequency (cyc/deg)
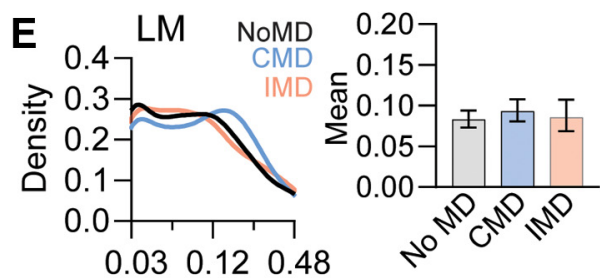

Spatial Frequency (cyc/deg)

\section{$\mathbf{H}$}
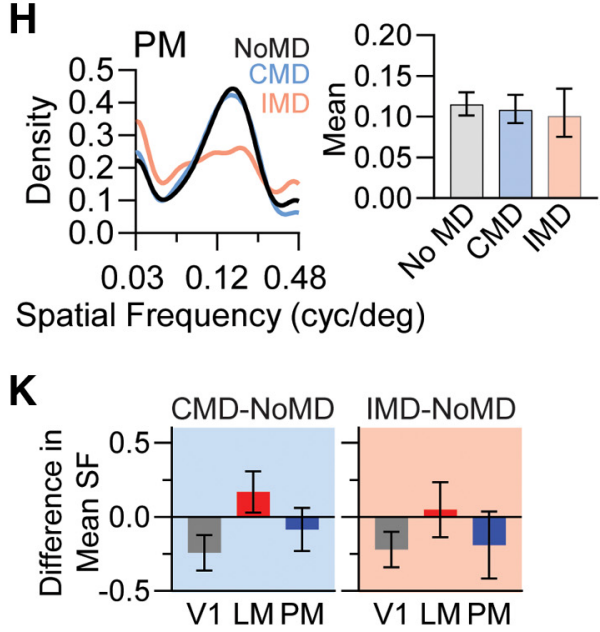
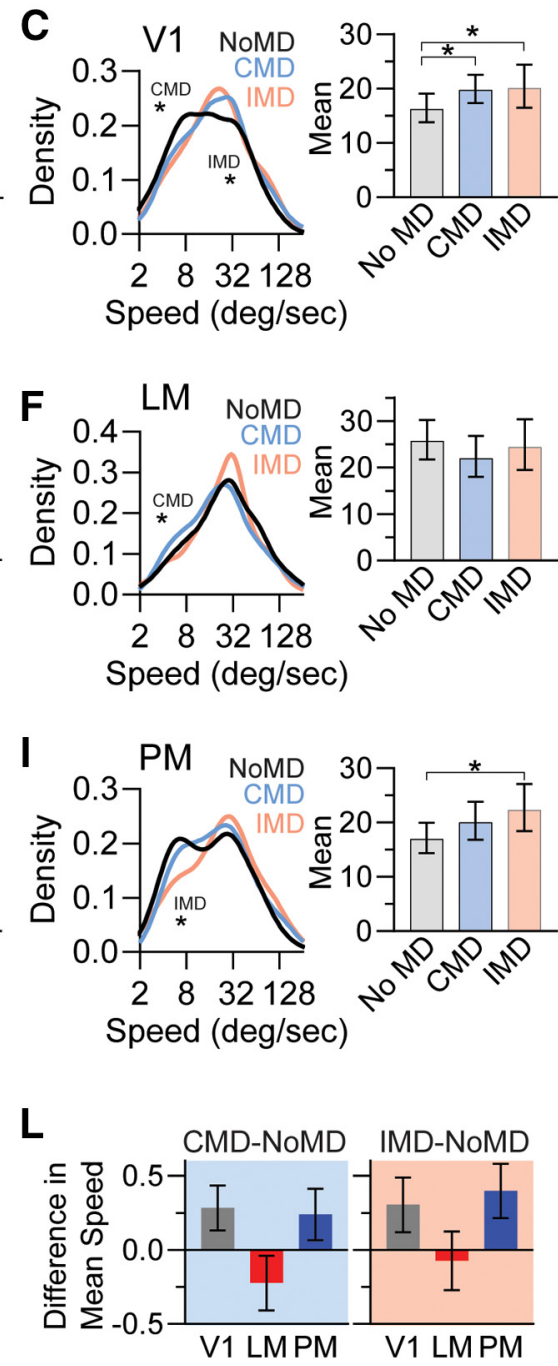

Figure 6. Monocular deprivation shifts spatiotemporal tuning differentially in V1, LM, and PM. $\boldsymbol{A}-\boldsymbol{C}, \mathrm{V} 1$ temporal frequency preferences $(\boldsymbol{A})$, spatial frequency preferences $(\boldsymbol{B})$, and speed preferences (C). Left, Density plots of preferred tuning of cells recorded from NoMD (black), CMD (blue), and IMD (pink) mice. Right, Bootstrapped means for preferred tuning of NoMD (gray), CMD (blue), and IMD (pink) mice. $\boldsymbol{D}-\boldsymbol{F}$, LM temporal frequency preferences (D), spatial frequency preferences $(\boldsymbol{E})$, and speed preferences $(\boldsymbol{F})$. Same conventions as in $\boldsymbol{A}-\boldsymbol{C}$ but for LM. $\mathbf{G}-\boldsymbol{I}$, PM temporal frequency preferences $(\boldsymbol{G})$, spatial frequency preferences $(\boldsymbol{H})$, and speed preferences $(\boldsymbol{I})$. Same convention as in $\boldsymbol{A}-\boldsymbol{C}$ but for PM. $\boldsymbol{J}$, Difference in octaves in the bootstrapped mean preferred temporal frequency of NoMD and CMD mice (left, shaded blue) or NoMD and IMD mice (right, shaded pink) for V1 (gray), LM (red), and PM (blue). $K$, Difference in the bootstrapped mean preferred spatial frequency of NoMD and CMD mice or NoMD and IMD mice (same convention as $\boldsymbol{J}$ ). $L$, Difference in the bootstrapped mean preferred speed of NoMD and CMD mice or NoMD and IMD mice (same convention as $\boldsymbol{J}$ ). Error bars in $A-I$ represent confidence intervals. Error bars in $J-L$ represent the SE for the difference of the means. For density plots, ${ }^{*}$ represents statistical significance with multiple-comparisons correction $(p<0.05 / 3)$. For bootstrapped mean plots, ${ }^{*} p<0.05$.

regardless of which eye was deprived. Second, we find reorganization in the functional specialization of eye-specific speed preferences within these areas. Third, despite changes in the relationship between ODI and the degree of speed tuning in V1, MD does not prevent the emergence of speed-tuned cells in LM and PM. Together, our findings indicate that binocular vision is required for the development of spatiotemporal tuning preferences among multiple visual areas, and of eye-specific spatiotemporal signals within individual areas.

While it has been shown that the functional differentiation of HVAs is refined over the course of development (Murakami et al., 2017), the role of visual experience in HVA differentiation has been unexplored. Our study demonstrates that MD during the ocular dominance critical period alters the spatiotemporal responses in mouse visual cortex, resulting in a reduction of areal differentiation that persists into adulthood after binocular vision is restored (Fig. 10). It has been proposed that areas of the putative dorsal stream take longer to develop than areas of the putative ventral stream (Smith et al., 2017). Here, we show that speed preferences of area LM, which is considered to belong to the putative ventral stream, is altered with $\mathrm{MD}$, particularly CMD (Fig. 6F), while the selectivity of PM, grouped into the putative dorsal stream, is disrupted more by IMD (Fig. 6I). In contrast, both CMD and IMD led to the same shift of speed preferences toward faster speeds in V1 (Fig. 6C). The shifts in speed preferences were opposite in direction for LM and PM, revealing that the HVAs were not simply inheriting changes that occur at the level of V1 (Fig. 6J-L). Together, these results suggest that proper binocular visual experience, and not just proper contralateral eye visual experience, is required for the functional differentiation of HVAs.

Here, we used single-cell resolution imaging and probed both contralateral and ipsilateral eye stimulation in V1 and HVAs to reveal that the differentiation of tuning selectivity among these areas is reduced by both CMD and IMD during the ocular dominance critical period. Although we have not directly 


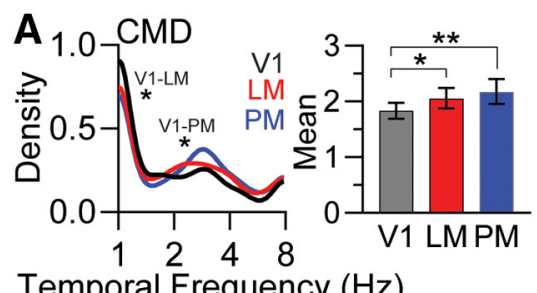

Temporal Frequency $(\mathrm{Hz})$

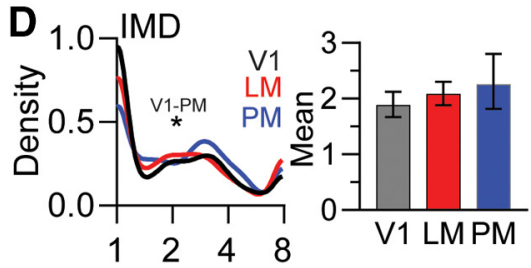

Temporal Frequency $(\mathrm{Hz})$

G
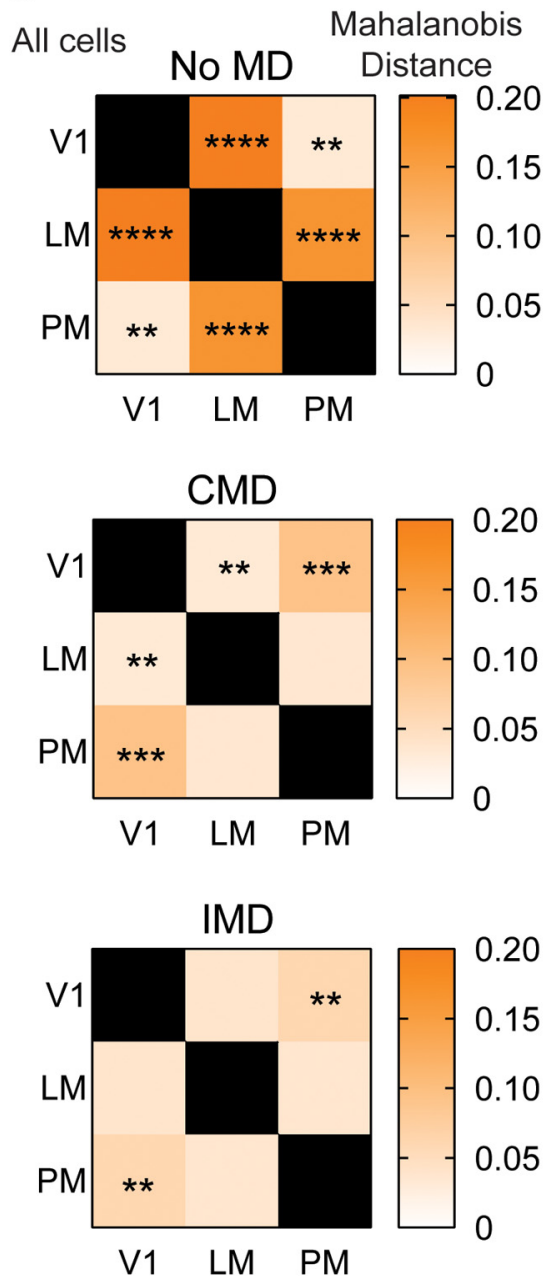
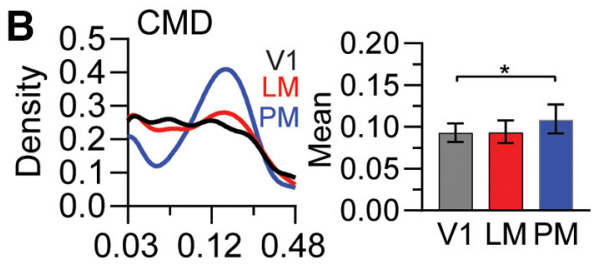

Spatial Frequency (cyc/deg)

E

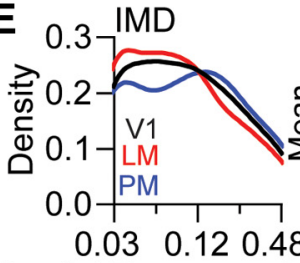

0.20

0.15

$\sum_{0.05-1}^{\mathbb{\Phi}} 0.10-$

0.00

$$
0.03 \quad 0.120 .48
$$

Spatial Frequency (cyc/deg)

\section{H}

Matched by animal

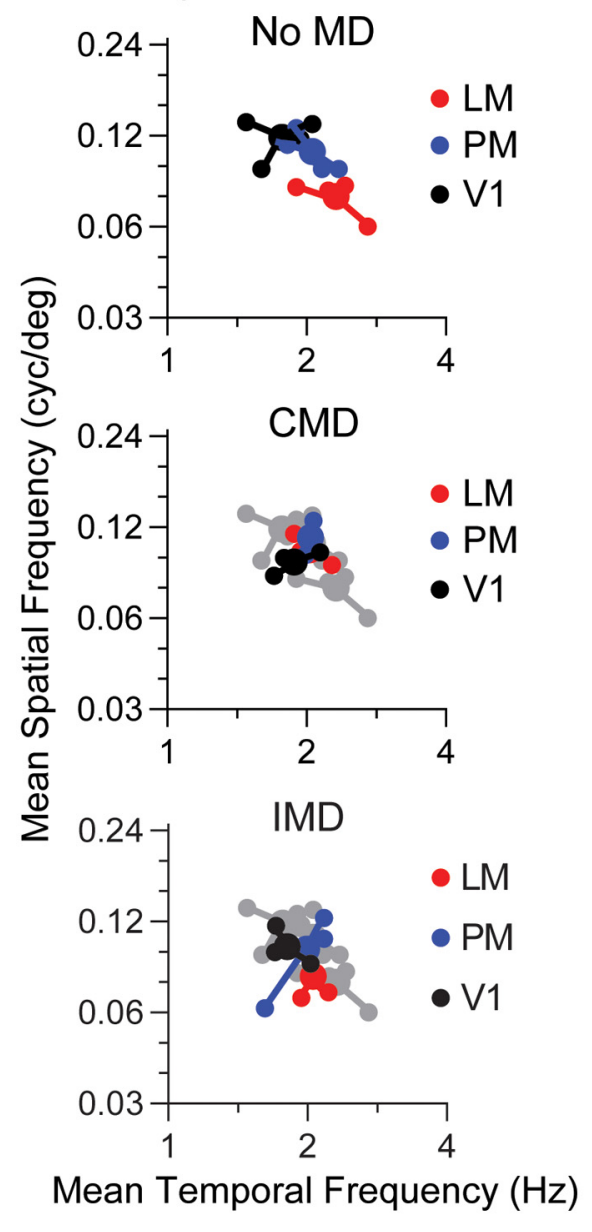

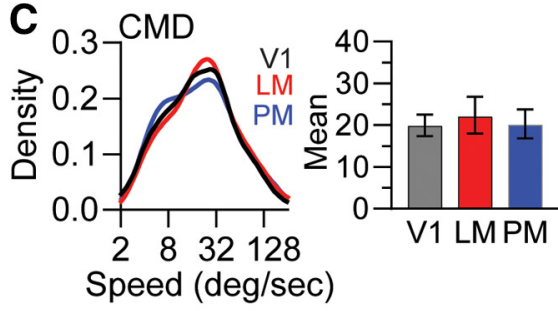

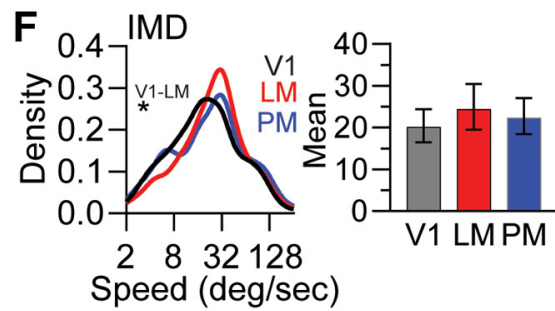

I

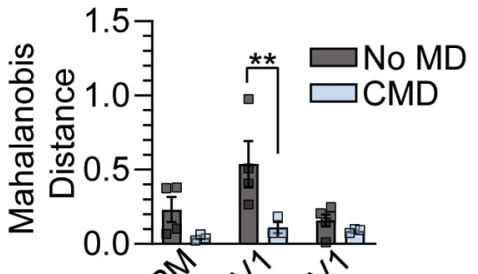

Figure 7. Monocular deprivation disrupts proper functional segregation of higher visual areas. $\boldsymbol{A}-\boldsymbol{C}$, Preferred temporal frequency $(\boldsymbol{A})$, spatial frequency $(\boldsymbol{B})$, and speed $(\boldsymbol{C})$ of cells recorded in CMD mice. Left, Density plots of preferred tuning of cells in V1 (black), LM (red), and PM (blue). Right, Bootstrapped means for preferred tuning of cells in V1 (black), LM (red), and PM (blue). $\boldsymbol{D}-\boldsymbol{F}$, Preferred temporal frequency $(\boldsymbol{D})$, spatial frequency $(\boldsymbol{E})$, and speed $(\boldsymbol{F})$ of cells recorded in IMD mice. Same convention as in $\boldsymbol{A}-\boldsymbol{C}$ but for cells recorded in IMD mice. $\boldsymbol{G}$, Heatmap of interareal Mahalanobis distances in NoMD (top), CMD (middle), and IMD (bottom) mice. $\boldsymbol{H}$, Scatter plot for mean preferred spatial frequency and mean preferred temporal frequency for V1, LM, and PM in matched NoMD (top), CMD (middle), or IMD (bottom) mice. Each individual point is an animal while larger points represent the mean of the means. Control data are shown in gray. I, Top, Matched by animal mean Mahalanobis distances for interareal comparisons in NoMD (gray) and CMD (blue) mice. Bottom, Matched by animal mean summed Mahalanobis distance for interareal comparisons in NoMD and CMD mice. J, Top, Matched by animal interareal Mahalanobis distances for NoMD (gray) and IMD (pink) mice. Bottom, Matched by animal mean summed interareal Mahalanobis distances for NoMD and IMD mice. For $\boldsymbol{A}-\boldsymbol{F}$, error bars represent confidence intervals. For $\boldsymbol{I}$ and $\boldsymbol{J}$, error bars represent the $\mathbf{S E M}$. For $\boldsymbol{I}$ and $\boldsymbol{J}$ each individual point represents data from one animal. For density plots in $\boldsymbol{A}-\boldsymbol{F},{ }^{*}$ represents statistical significance with multiple-comparisons correction $(p<0.05 / 3)$. For bootstrapped mean plots in $\boldsymbol{A}-\boldsymbol{F}$, and for panels $\mathbf{G}$ and $\boldsymbol{I}$, ${ }^{*} p<0.05 ;{ }^{* *} p<0.01$; ${ }^{* * *} p<0.001$; ${ }^{* * * *} p<0.0001$. 
Table 4. Visual properties in CMD mice

\begin{tabular}{|c|c|c|c|c|c|c|c|c|c|}
\hline Figure panel & Measure & V1 & LM & PM & Statistic & By cell or by animal & V1 vs LM & V1 vs PM & LM vs PM \\
\hline gure $7 A-C$ & $\begin{array}{l}\text { Sample size: \# neurons } \\
\text { (\# mice) }\end{array}$ & 726 (6 mice) & 567 (4 mice) & 216 (4 mice) & & & & & \\
\hline Figure $7 A$, left & TF median $(\mathrm{Hz})$ & 1.4 & 1.91 & 2.21 & KW test ${ }^{*}, p=0.0003, \mathrm{KW}(\mathrm{s})=16.5$ & By cell & $p=0.0054$ & $p=0.0016$ & NS, $p=0.74$ \\
\hline Figure $7 A$, right & TF mean (Hz) & 1.83 & 2.05 & 2.17 & Hierarchical bootstrap & By cell & $p=0.031$ & $p=0.0047$ & NS, $p=0.10$ \\
\hline Figure $7 B$, left & SF median $\left(\right.$ cycle $\left./^{\circ}\right)$ & 0.088 & 0.096 & 0.12 & KW test ${ }^{*}, \mathrm{NS}, p=0.034$ & By cell & NS, $p>0.99$ & $N S, p=0.033$ & NS, $p=0.07$ \\
\hline Figure $7 B$, right & SF mean $\left(\right.$ cycle $\left./^{\circ}\right)$ & 0.093 & 0.093 & 0.11 & Hierarchical bootstrap & By cell & NS, $p=0.53$ & $p=0.045$ & NS, $p=0.08$ \\
\hline igure $7 C$, left & Speed median $(\% / s)$ & 19.4 & 22.2 & 21.1 & KW test ${ }^{*}, \mathrm{NS}, p=0.18$ & By cell & NS, $p=0.24$ & NS, $p>0.99$ & NS, $p=0.68$ \\
\hline Figure $7 C$, right & Speed mean ( $\%$ s) & 19.8 & 22 & 20 & Hierarchical bootstrap & By cell & NS, $p=0.18$ & NS, $p=0.45$ & NS, $p=0.24$ \\
\hline
\end{tabular}

KW, Kruskal-Wallis. TF, SF, and TF/SF preferences of cells that were visually responsive in contralateral eye-deprived mice.

*Statistics with a multiple-comparisons correction.

Table 5. Visual properties in IMD mice

\begin{tabular}{|c|c|c|c|c|c|c|c|c|c|}
\hline Figure panel & Measure & V1 & LM & PM & Statistic & By cell or by animal & V1 vs LM & V1 vs PM & LM vs PM \\
\hline Figure $7 D-F$ & $\begin{array}{l}\text { Sample size: \# neurons } \\
\text { (\# mice) }\end{array}$ & 357 (4 mice) & 370 (4 mice) & 202 (4 mice) & & & & & \\
\hline Figure $7 D$, left & TF median (Hz) & 1.67 & 1.88 & 2.21 & KW test ${ }^{*}, p=0.0072, \mathrm{KW}(\mathrm{s})=9.8$ & By cell & NS, $p=0.12$ & $p=0.007$ & $\mathrm{NS}, p=0.57$ \\
\hline Figure $7 D$, right & TF mean $(\mathrm{Hz})$ & 1.88 & 2.08 & 2.25 & Hierarchical bootstrap & By cell & NS, $p=0.098$ & NS, $p=0.072$ & NS, $p=0.26$ \\
\hline Figure $7 E$, left & SF median $\left(\right.$ cycle $\left./{ }^{\circ}\right)$ & 0.091 & 0.078 & 0.11 & KW test ${ }^{*}, N S, p=0.096$ & By cell & NS, $p=0.41$ & NS, $p>0.99$ & NS, $p=0.12$ \\
\hline Figure $7 E$, right & SF mean $\left(\right.$ cycle $\left./^{\circ}\right)$ & 0.094 & 0.086 & 0.1 & Hierarchical bootstrap & By cell & NS, $p=0.24$ & NS, $p=0.32$ & NS, $p=0.19$ \\
\hline Figure $7 F$, left & Speed median ( $\% / s)$ & 20.4 & 28.3 & 25.3 & KW test ${ }^{*}, p=0.013, \mathrm{KW}(\mathrm{s})=8.7$ & By cell & $p=0.0095$ & NS, $p=0.47$ & NS, $p=0.85$ \\
\hline Figure $7 F$, right & Speed mean ( $\%$ s) & 20.1 & 24.4 & 22.3 & Hierarchical bootstrap & By cell & NS, $p=0.093$ & NS, $p=0.22$ & NS, $p=0.27$ \\
\hline
\end{tabular}

KW, Kruskal-Wallis. TF, SF, and TF/SF preferences of cells that were visually responsive in ipsilateral eye-deprived mice.

*Statistics with a multiple-comparisons correction.

Table 6. Interareal Mahalanobis distance analysis

\begin{tabular}{|c|c|c|c|c|c|c|}
\hline Figure panel & Measure/statistic & Area & NoMD & CMD & IMD & By cell or by animal \\
\hline \multirow[t]{3}{*}{ Figure $7 G$} & \multirow[t]{3}{*}{ Sample size: \# neurons (\# mice) } & V1 & 819 (8 mice) & 726 (6 mice) & 357 (4 mice) & \\
\hline & & LM & 645 (7 mice) & 567 (4 mice) & 370 (4 mice) & \\
\hline & & PM & 488 (8 mice) & 216 (4 mice) & 202 (4 mice) & \\
\hline \multirow[t]{3}{*}{ Figure $7 G$} & \multirow[t]{3}{*}{ Mahalanobis distance, all cells } & V1 vs LM & 0.202 & 0.033 & 0.04 & By cell \\
\hline & & V1 vs PM & 0.033 & 0.093 & 0.076 & By cell \\
\hline & & LM vs PM & 0.167 & 0.036 & 0.043 & By cell \\
\hline \multirow[t]{3}{*}{ Figure $7 G$} & \multirow[t]{3}{*}{ Wilk's $\lambda$ test $^{*}$ on Mahalanobis distance } & V1 vs LM & $p<0.0001$ & $p=0.0058$ & NS, $p=0.027$ & By cell \\
\hline & & V1 vs PM & $p=0.0064$ & $p=0.0004$ & $p=0.008$ & By cell \\
\hline & & LM vs PM & $p<0.0001$ & NS, $p=0.058$ & NS, $p=0.062$ & By cell \\
\hline \multirow[t]{3}{*}{ Figure $7 /-J$, top } & \multirow[t]{3}{*}{ Mean Mahalanobis distance, by animal } & V1 vs LM & 0.54 & 0.11 & 0.15 & By animal ${ }^{\#}$ \\
\hline & & V1 vs PM & 0.16 & 0.089 & 0.28 & By animal $^{\#}$ \\
\hline & & LM vs PM & 0.23 & 0.043 & 0.2 & By animal $^{\#}$ \\
\hline Figure $7 /, J$, bottom & Summed Mahalanobis distance & All pairwise & 0.92 & 0.24 & 0.65 & By animal \\
\hline \multirow[t]{4}{*}{ Figure 71, top } & $\begin{array}{l}\text { Two-way ANOVA on mean Mahalanobis } \\
\text { distance }\end{array}$ & $\begin{array}{l}\text { NoMD vs CMD: area } p=0.06 \text {; deprivation } p=0.022 ; \\
\quad \text { area } x \text { deprivation } p=0.14\end{array}$ & & & & \\
\hline & \multirow[t]{3}{*}{ Sidak's post hoc test } & V1 vs LM (No MD vs CMD) & $p=0.0091$ & & & \\
\hline & & V1 vs PM (No MD vs CMD) & NS, $p=0.91$ & & & \\
\hline & & LM vs PM (No MD vs CMD) & NS, $p=0.37$ & & & \\
\hline Figure 71 , bottom & Welch's unpaired $t$ test & No MD vs CMD & $p=0.039$ & & & \\
\hline \multirow[t]{4}{*}{ Figure $7 J$, top } & $\begin{array}{l}\text { Two-way ANOVA on mean Mahalanobis } \\
\text { distance }\end{array}$ & $\begin{array}{l}\text { NoMD vs IMD: area } p=0.40 \text {; deprivation } p=0.28 \\
\quad \text { area } \mathrm{x} \text { deprivation } p=0.078\end{array}$ & & & & \\
\hline & \multirow[t]{3}{*}{ Sidak's post hoc test } & V1 vs LM (No MD vs IMD) & $p=0.042$ & & & \\
\hline & & V1 vs PM (No MD vs IMD) & NS, $p=0.82$ & & & \\
\hline & & LM vs PM (No MD vs IMD) & NS, $p=0.99$ & & & \\
\hline Figure 71 , bottom & Welch's unpaired $t$ test & No MD vs IMD & $p=0.38$ & & & \\
\hline
\end{tabular}

Mahalanobis distances based on preferred spatial and temporal frequency for each area in NoMD, CMD, and IMD mice.

* Statistics with a multiple-comparisons correction.

${ }^{\#} \mathrm{~N}=4$ for NoMD mice and $\mathrm{N}=3$ for CMD or IMD mice.

demonstrated that MD results in a disruption of development rather than a deterioration of some functional properties (by depriving the mice of visual input after the higher visual areas have reached adult-like patterns at $\sim$ P25, based on the study by Murakami et al., 2017), we hypothesize that MD disrupts development for the following three reasons: (1) we failed to find a difference in the percentage of cells that were visually driven following $\mathrm{MD}$, suggesting a shift in spatiotemporal tuning rather than a deterioration; (2) the spatiotemporal tuning of eye-specific responses suggests that binocular cell preferences are shifting in V1; and (3) behaviorally measured visual acuity refines well after $\mathrm{P} 25$, up until P40, and long-term MD (P24-P45) results in a 

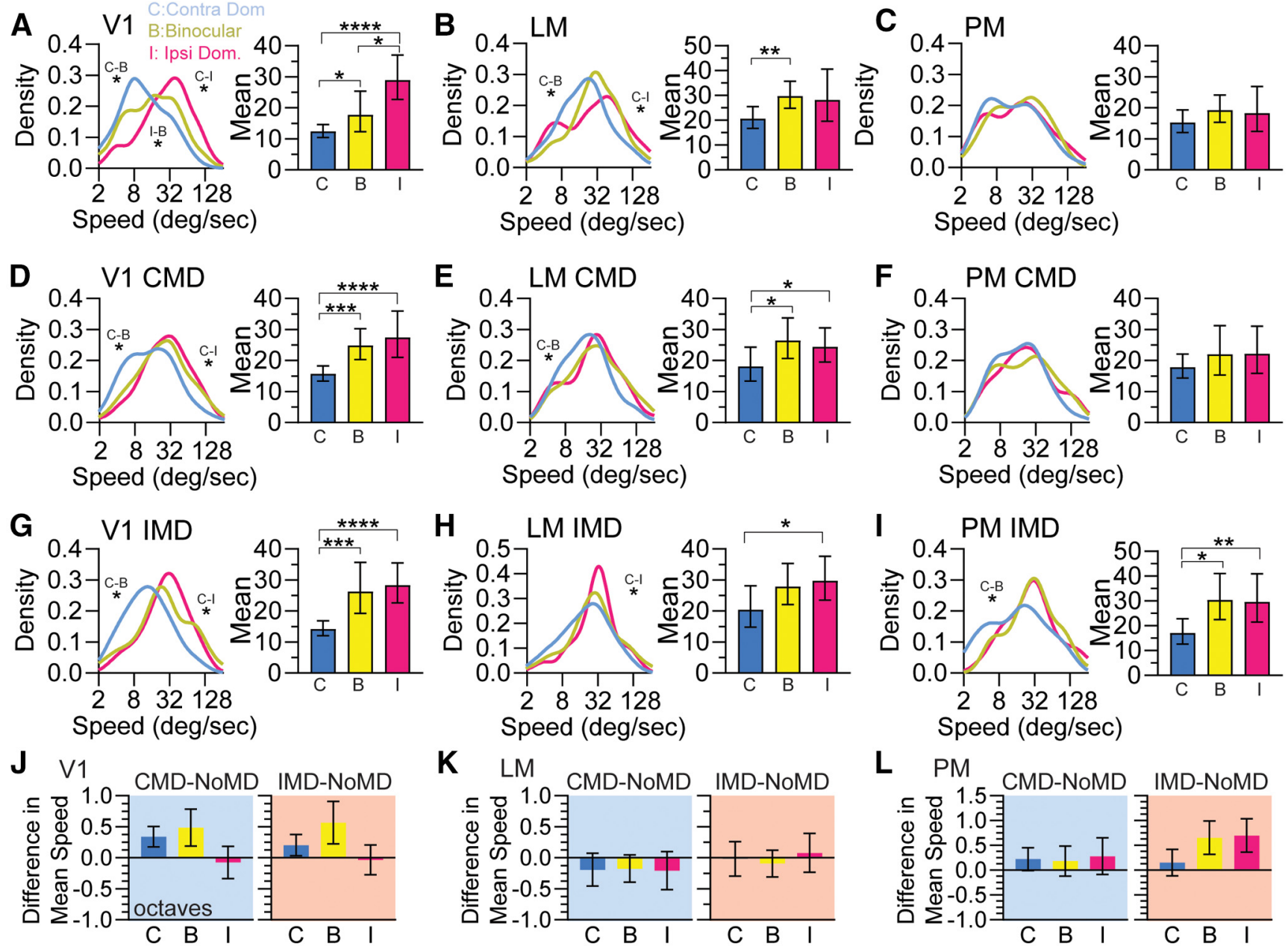

M
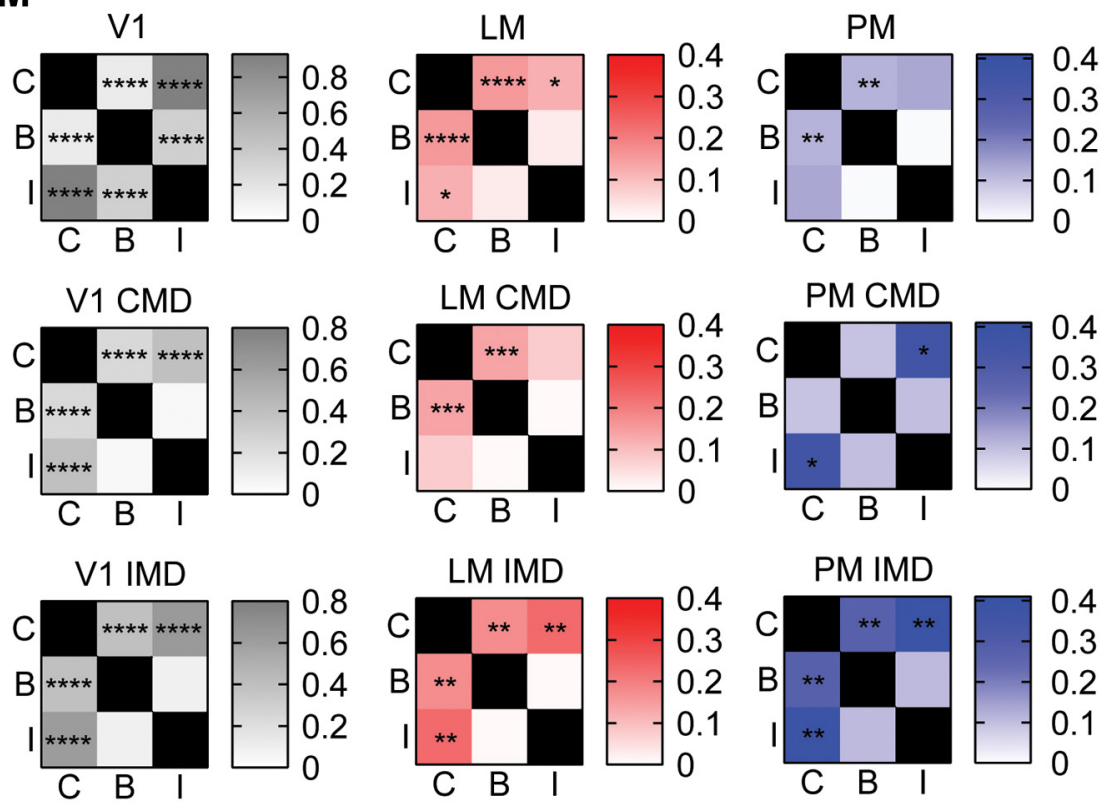

\section{PM CMD-NoMD IMD-NoMD}

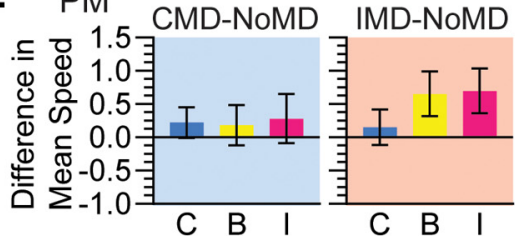

$\mathbf{N}$
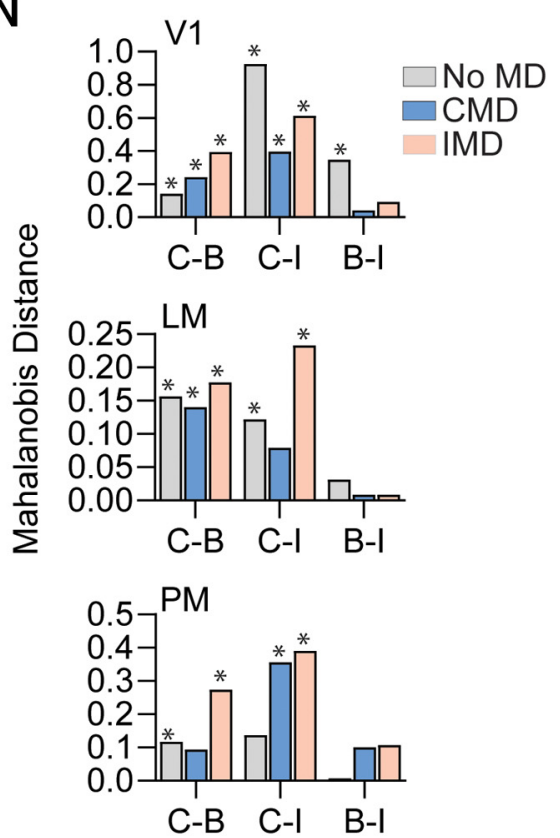

Figure 8. Monocular deprivation disrupts proper functional segregation of eye-specific responses in visual cortex. $\boldsymbol{A}-\boldsymbol{C}$, Preferred speed of eye-specific responses recorded in V1 $(\boldsymbol{A})$, LM (B), and PM $(C)$ of NoMD mice. Left, Density plots of preferred speeds of eye-specific responses (blue, contralateral dominated; yellow, binocular; pink, ipsilateral dominated) from NoMD mice. Right, Bootstrapped means for preferred speeds of eye-specific responsive cells (blue, contralateral dominated; yellow, binocular; pink, ipsilateral dominated) from NoMD mice. $\boldsymbol{D}-\boldsymbol{F}$, Preferred speed of eye-specific responses recorded in V1 $(\boldsymbol{D})$, LM $(\boldsymbol{E})$, and PM $(\boldsymbol{F})$ of CMD mice. Same convention as in $\boldsymbol{A}-\boldsymbol{C}$ but for cells recorded in CMD mice. $\mathbf{G}-\boldsymbol{I}$, Preferred speed of eye-specific responses recorded in V1 $(\boldsymbol{G})$, LM $(\boldsymbol{H})$, and PM $(\boldsymbol{I})$ of IMD mice. Same convention as in $\boldsymbol{A}-\boldsymbol{C}$ but for cells recorded in IMD mice. $\boldsymbol{J}-\boldsymbol{L}$, Difference in octaves in the bootstrapped mean preferred speed of eye-specific responses in NoMD and CMD mice (left, shaded blue) or NoMD and IMD mice (right, shaded pink) for V1 (J), LM (K), and PM (L). $M$, Heatmaps for intra-areal eye-specific Mahalanobis distances for preferred spatiotemporal frequency in V1 (left, black column), LM (middle, red column), and PM (right, blue column) for NoMD (top row), CMD (middle row), and 
Table 7. Eye-specific visual properties in NoMD mice

\begin{tabular}{|c|c|c|c|c|c|c|c|c|c|c|}
\hline Figure panel & Measure & Area & $C$ & $B$ & I & Statistic & By cell or by animal & C vs $B$ & C vs I & B vs I \\
\hline \multirow[t]{3}{*}{ Figure $8 A-C$, all } & $\begin{array}{l}\text { Sample size: \# } \\
\text { neurons (\# mice) }\end{array}$ & V1 & 418 (8 mice) & 226 (8 mice) & 174 (8 mice) & & & & & \\
\hline & & LM & 248 (7 mice) & 296 (7 mice) & 101 (7 mice) & & & & & \\
\hline & & PM & 245 (8 mice) & 175 (8 mice) & 68 (8 mice) & & & & & \\
\hline \multirow[t]{3}{*}{ Figure $8 A-C$, left } & Speed median ( $\%$ s) & V1 & 11.3 & 18.1 & 33.3 & $\begin{array}{c}\mathrm{KW} \text { test }^{*}, p<0.0001, \\
\mathrm{KW}(\mathrm{s})=85.2\end{array}$ & By cell & $p=0.0002$ & $p<0.0001$ & $p<0.0001$ \\
\hline & & $L M$ & 20 & 32.9 & 33.2 & $\begin{array}{c}\text { KW test }{ }^{*}, p<0.0001, \\
K W(s)=22.3\end{array}$ & By cell & $p<0.0001$ & $p=0.013$ & NS, $p>0.99$ \\
\hline & & PM & 14.2 & 22 & 19.2 & $\begin{array}{c}\text { KW test }{ }^{*}, p=0.04, \\
\mathrm{KW}(\mathrm{s})=6.3\end{array}$ & By cell & $p=0.039$ & NS, $p=0.77$ & NS, $p>0.99$ \\
\hline \multirow[t]{3}{*}{ Figure $8 A-C$, right } & Speed mean $(\% / s)$ & V1 & 12.4 & 17.7 & 28.9 & Hierarchical bootstrap & By cell & $p=0.029$ & $p<0.0001$ & $p=0.12$ \\
\hline & & LM & 20.6 & 29.8 & 28.2 & Hierarchical bootstrap & By cell & $p=0.006$ & $p=0.071$ & $p=0.41$ \\
\hline & & PM & 15.2 & 19.3 & 18.3 & Hierarchical bootstrap & By cell & NS, $p=0.071$ & NS, $p=0.21$ & NS, $p=0.42$ \\
\hline
\end{tabular}

KW, Kruskal-Wallis. Preferred speed preferences for eye-specific responses in each area for control mice.

* Statistics with a multiple-comparisons correction.

Table 8. Eye-specific visual properties in CMD mice

\begin{tabular}{|c|c|c|c|c|c|c|c|c|c|c|}
\hline Figure panel & Measure & Area & $\mathrm{C}$ & $B$ & $\mathrm{I}$ & Statistic & By cell or by animal & C vs $B$ & C vs I & B vs I \\
\hline \multirow[t]{3}{*}{ Figure $8 D-F$, all } & $\begin{array}{l}\text { Sample size: \# } \\
\text { neurons (\# mice) }\end{array}$ & V1 & 391 (6 mice) & 174 (6 mice) & 161 (6 mice) & & & & & \\
\hline & & LM & 246 (4 mice) & 193 (4 mice) & 128 (4 mice) & & & & & \\
\hline & & PM & 96 (4 mice) & 81 (4 mice) & 39 (4 mice) & & & & & \\
\hline \multirow[t]{3}{*}{ Figure $8 D-F$, left } & Speed median $(\% / s)$ & V1 & 16.5 & 27.9 & 31.2 & $\begin{array}{c}\text { KW test }{ }^{*}, p<0.0001, \\
K W(s)=47.1\end{array}$ & By cell & $p<0.0001$ & $p<0.0001$ & NS, $p=0.63$ \\
\hline & & LM & 18 & 26.4 & 25.8 & $\begin{array}{c}\text { KW test }{ }^{*}, p=0.0005 \\
\mathrm{KW}(\mathrm{s})=15.4\end{array}$ & By cell & $p=0.0005$ & $p=0.03$ & NS, $p>0.99$ \\
\hline & & PM & 17.5 & 23.6 & 21.5 & $\begin{array}{l}\text { KW test }{ }^{*}, p=0.34 \\
K W(s)=2.1\end{array}$ & By cell & NS, $p=0.52$ & NS, $p=0.95$ & NS, $p>0.99$ \\
\hline \multirow[t]{3}{*}{ Figure $8 D-F$, right } & Speed mean $(\% / s)$ & V1 & 15.7 & 24.8 & 27.5 & Hierarchical bootstrap & By cell & $p=0.0002$ & $p<0.0001$ & NS, $p=0.25$ \\
\hline & & LM & 18.1 & 26.5 & 24.4 & Hierarchical bootstrap & By cell & $p=0.017$ & $p=0.04$ & NS, $p=0.31$ \\
\hline & & PM & 17.8 & 21.9 & 22.2 & Hierarchical bootstrap & By cell & NS, $p=0.15$ & NS, $p=0.13$ & NS, $p=0.52$ \\
\hline
\end{tabular}

KW, Kruskal-Wallis. Preferred speed preferences for eye-specific responses in each area for contralateral eye-deprived mice.

* Statistics with a multiple-comparisons correction.

Table 9. Eye-specific visual properties in IMD mice

\begin{tabular}{|c|c|c|c|c|c|c|c|c|c|c|}
\hline Figure panel & Measure & Area & $C$ & B & $\mathrm{I}$ & Statistic & By cell or by animal & C vs $B$ & C vs I & B vs I \\
\hline \multirow[t]{3}{*}{ Figure $8 G-I$, all } & $\begin{array}{l}\text { Sample size: \# } \\
\text { neurons (\# mice) }\end{array}$ & V1 & 162 (4 mice) & 124 (4 mice) & 71 (4 mice) & & & & & \\
\hline & & LM & 171 (4 mice) & 132 (4 mice) & 67 (4 mice) & & & & & \\
\hline & & PM & 105 (4 mice) & 51 (4 mice) & 46 (4 mice) & & & & & \\
\hline \multirow[t]{3}{*}{ Figure $8 G-l$, left } & Speed median ( $\% / s)$ & V1 & 13.9 & 26.6 & 33.2 & $\begin{array}{c}\text { KW test }{ }^{*}, p<0.0001 \\
K W(s)=39.8\end{array}$ & By cell & $p<0.0001$ & $p<0.0001$ & NS, $p>0.99$ \\
\hline & & LM & 21.9 & 32.6 & 33.3 & $\begin{array}{c}\text { KW test* }, p=0.003 \\
\text { KW(s) }=11.6\end{array}$ & By cell & $p=0.023$ & $p=0.011$ & NS, $p>0.99$ \\
\hline & & PM & 18.3 & 33.3 & 30.9 & $\begin{array}{l}\mathrm{KW} \text { test }{ }^{*}, p=0.0028 \\
\mathrm{KW}(\mathrm{s})=11.7\end{array}$ & By cell & $p=0.009$ & $p=0.031$ & NS, $p>0.99$ \\
\hline \multirow[t]{3}{*}{ Figure $8 G-I$, right } & Speed mean $(\% / s)$ & V1 & 14.2 & 26.2 & 28.4 & Hierarchical bootstrap & By cell & $p=0.0005$ & $p<0.0001$ & NS, $p=0.33$ \\
\hline & & LM & 20.4 & 27.8 & 29.8 & Hierarchical bootstrap & By cell & NS, $p=0.06$ & $p=0.029$ & NS, $p=0.66$ \\
\hline & & PM & 17 & 30.4 & 29.6 & Hierarchical bootstrap & By cell & $p=0.002$ & $p=0.005$ & NS, $p=0.46$ \\
\hline
\end{tabular}

KW, Kruskal-Wallis. Preferred speed preferences for eye-specific responses in each area for ipsilateral eye-deprived mice.

*Statistics with a multiple-comparisons correction.

\footnotetext{
$\leftarrow$

IMD (bottom row) mice. $\boldsymbol{N}$, Summary bar plot for Mahalanobis distances shown in $\boldsymbol{M}$. For $\boldsymbol{A -}$ $\boldsymbol{I}$, error bars represent confidence intervals. For density plots in $\boldsymbol{A}-\boldsymbol{I},{ }^{*}$ represents statistical significance with multiple-comparisons correction $(p<0.05 / 3)$. Error bars in $\boldsymbol{J}-\boldsymbol{L}$ represent the SE for the difference of the means. For bootstrapped mean plots in $\boldsymbol{A} \boldsymbol{I} \boldsymbol{I}$ and panel $\boldsymbol{M}$, ${ }^{*} p<0.05 ;{ }^{* *} p<0.01 ;{ }^{* * *} p<0.001 ;{ }^{* * * *} p<0.0001$. For $\boldsymbol{N}$, * indicates any statistically significant comparison found in $\boldsymbol{M}(p<0.05)$.
}

permanent deficit in acuity (Stephany et al., 2014). To address the mechanism by which MD results in a reduced differentiation of visual areas, one could track the response properties of single cells from different visual areas before and after deprivation during the ocular dominance critical period. Since Murakami et al. (2017) reported that anatomically defined dorsal stream areas may be further divided into multiple substreams, future studies could investigate whether the effects of MD are distinct for different putative dorsal stream areas. 
Table 10. Eye-specific Mahalanobis distance analysis

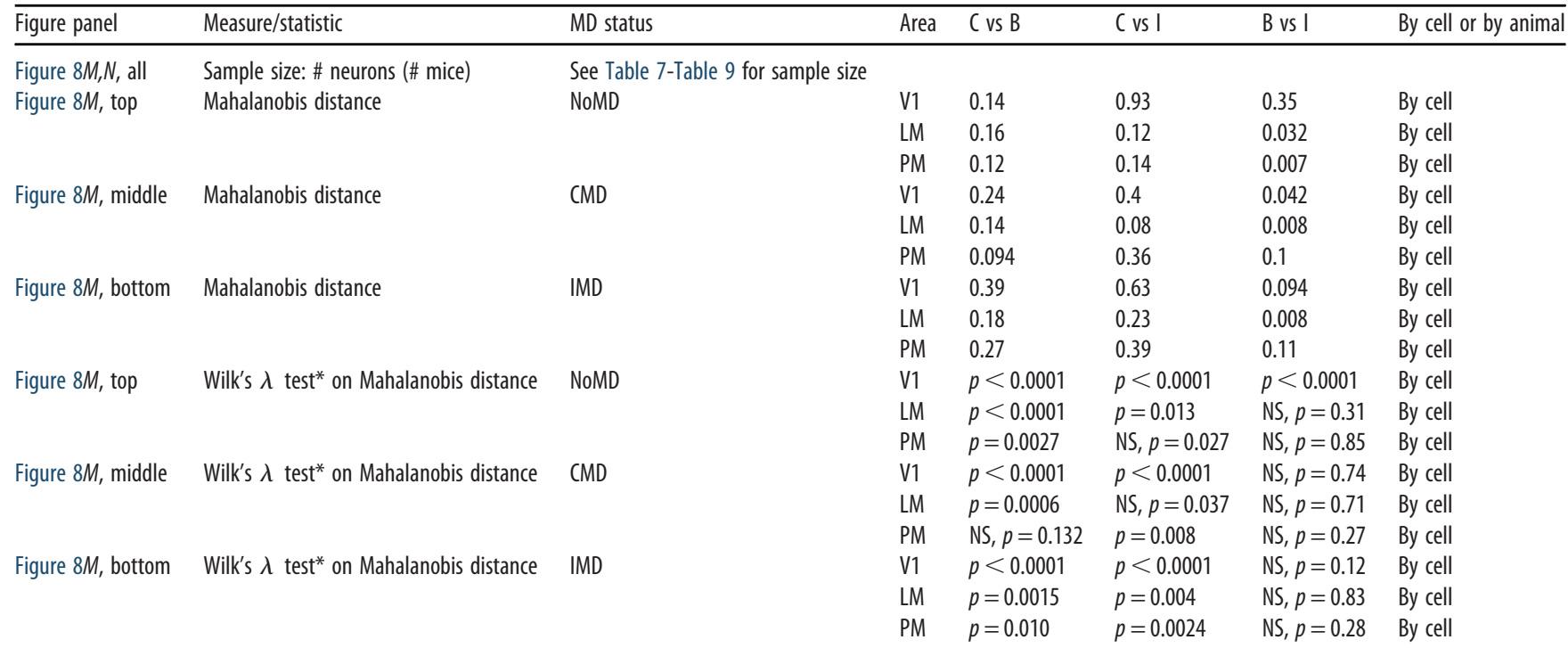

Mahalanobis distances based on preferred spatial and temporal frequency for eye-specific responses within each area in NoMD, CMD, and IMD mice.

*Statistics with a multiple-comparisons correction.
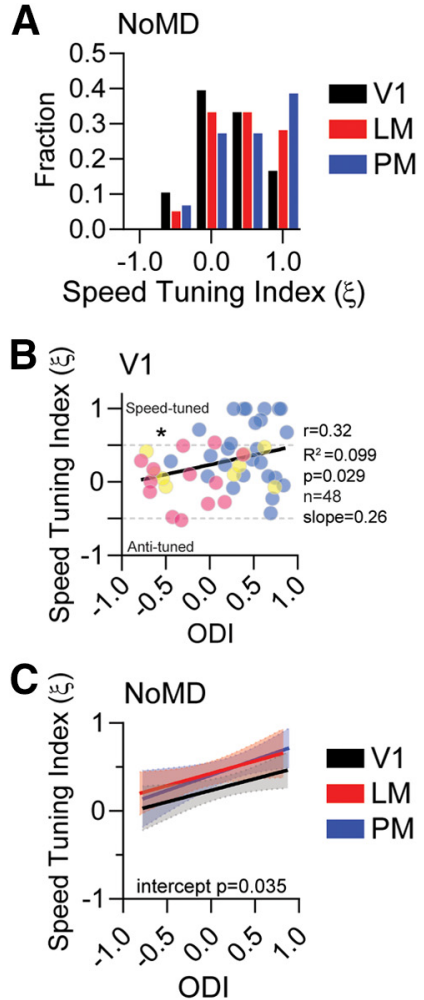
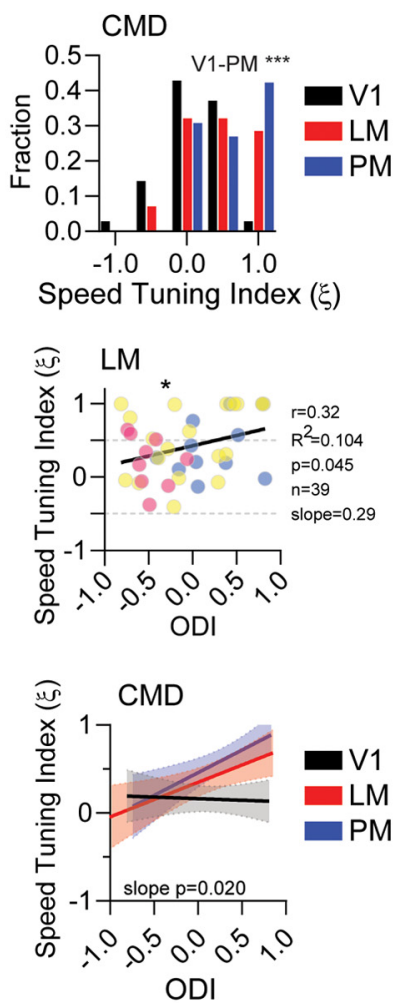
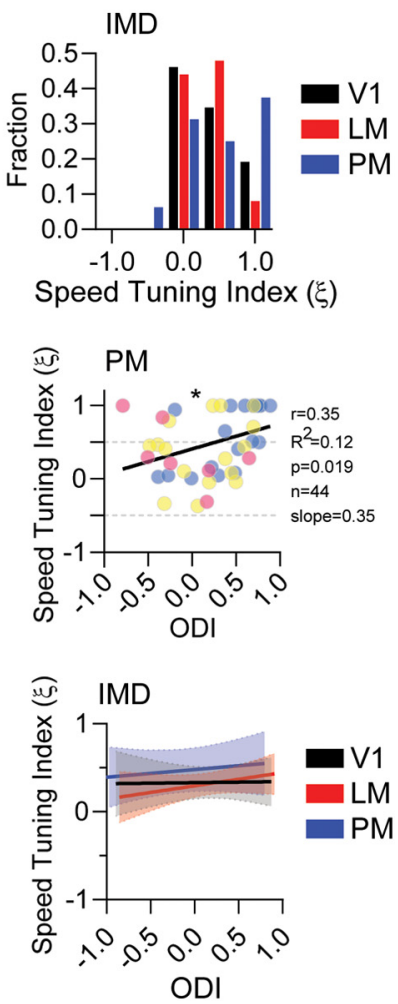

Figure 9. Monocular deprivation has distinct effects on the relationship between ocularity and speed tuning. $\boldsymbol{A}$, Speed-tuning indices of well fit cells for NoMD (left), CMD (middle), and IMD (right) mice in V1 (black), LM (red), and PM (blue). In CMD mice, the distributions of speed-tuning indices are significantly different. $\boldsymbol{B}$, Scatter plot of speed-tuning index against ODI color coded by eye specificity (contra dominated in blue, binocular in yellow and ipsi dominated in pink) in V1 (left), LM (middle), and PM (right) of NoMD mice. Linear regression is overlaid and correlations are specified at the right. C, Speed tuning versus ODI linear regressions are overlaid for V1 (black), LM (red), and PM (blue) for NoMD (left), CMD (middle), and IMD (right) mice. Shading corresponds to SE. For all ${ }^{*} p<0.05{ }^{* * *} p<0.001$.

Previously, we found that V1 cells dominated by the contralateral eye prefer higher spatial frequencies than binocular or ipsilateral dominated responses (Salinas et al., 2017). Here, we report that the spatiotemporal tuning is significantly correlated with ODI in V1 and that this relationship is less pronounced or absent in LM and PM in normally reared adult mice (Figs. 5, 8, 10). In V1, in the spatiotemporal domain, contralateral dominated responses are tuned to slower speeds (low temporal frequency/high spatial frequency), binocular cells are tuned to intermediate speeds, and ipsilateral dominated cells are tuned 
Table 11. Speed tuning index analysis

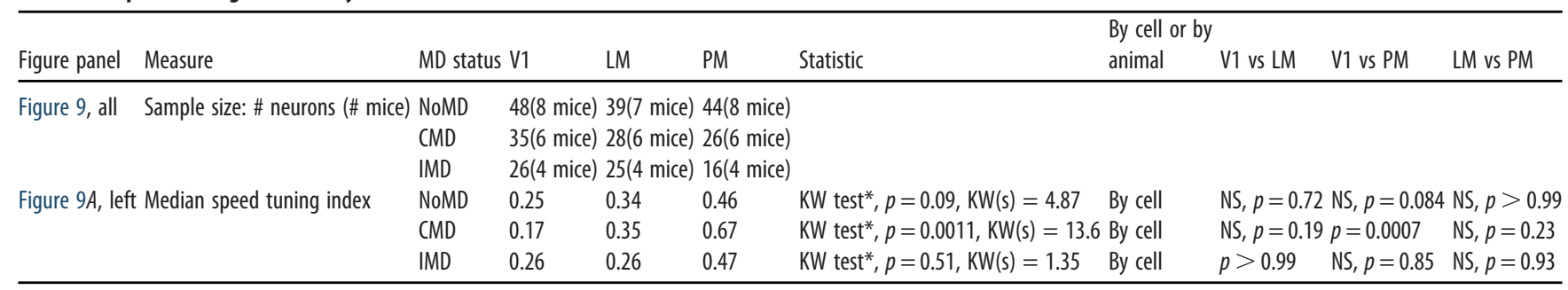

KW, Kruskal-Wallis. Speed-tuning indices, or the degree of speed tuning, for cells that passed the criteria for analysis for each area in NoMD, CMD, and IMD mice.

*Statistics with a multiple-comparisons correction.

to the fastest speeds (high temporal frequency/low spatial frequency). LM shares some of the eye-specific functional segregation found in $\mathrm{V} 1$, with the contralateral dominated cells tuned to the slowest speeds while PM is more homogenous in that regard. In control mice, eye-specific visual preferences are smaller in LM and $\mathrm{PM}$ than in V1, suggesting that better interocular matching of features emerges in downstream areas of cortex. MD (especially IMD) reverts LM and PM to a more eye-specific differentiated state (Figs. $5 D$, $8 J, 10)$, similar to $\mathrm{V} 1$, suggesting that binocular visual experience is required for proper interocular matching of spatiotemporal features in higher visual areas.

The functional specialization of visual areas may be supported by distinct subcortical inputs. Subregions of the dorsal lateral geniculate nucleus house functionally distinct cell types, which project to distinct layers of cortex (Krahe et al., 2011). For example, a subset of highly direction-selective and high spatial frequency-tuned cells of the shell region project to superficial layers of V1 (Cruz-Martín et al., 2014). Thus, the functional distinction of eye-specific responses in V1 and HVAs may be a result of distinct circuit pathways originating from the retina and flowing through distinct subcortical pathways (for review, see Seabrook et al., 2017). Consistent with this, we have recently demonstrated eye-specific spatial frequency tuning at the level of dorsal lateral geniculate nucleus inputs to V1 (Huh et al., 2020). The functionally distinct input from each eye may drive the organization and the functional architecture of V1 and HVAs.

The close association between ocular dominance and spatial frequency tuning in V1 has been demonstrated by numerous visual deprivation studies performed during the critical period for ocular dominance (Wiesel and Hubel, 1963; Dews and Wiesel, 1970; Hess and Howell, 1977; Levi and Harwerth, 1977; Fagiolini et al., 1994; Gordon and Stryker, 1996; Prusky and Douglas, 2003). Given the hierarchical model, which describes the receptive fields in HVAs as built on input from V1 cells, it would seem likely that MD would have compounding effects on HVA differentiation. In line with this prediction, amblyopic primates have severe deficits in the processing of higher-level feature detection, including contour integration, motion, and form processing (Kozma and Kiorpes, 2003; Kiorpes, 2006; Kiorpes et al., 2006). It has not been explored, however, whether the differentiation of HVAs into distinctly specialized functional modules requires binocular visual experience.
The formation of speed tuning in visual area MT (middle temporal) has been proposed to be a consequence of inputs from V1 neurons preferring the same speed but varying in their individual spatial and temporal frequency preferences (Heeger et al., 1996; Simoncelli and Heeger, 1998). Studies in macaque V1 and MT suggest that directionally selective V1 complex cells have the same degree of speed tuning as cells in area MT (Priebe et al., 2006). A hierarchical model would predict that the speed tuning of HVAs depends on spatiotemporal tuning of lower-level areas, namely V1 simple cells (Priebe et al., 2006). Surprisingly, we find that the degree of speed tuning of LM and PM is largely preserved following CMD (Figure 9). In contrast, speed preferences change in response to deprivation in all three areas (Figs. 6, 7).

While MD during the critical period reduces the functional differentiation of V1 and HVAs, it did not completely eliminate the degree of speed tuning in LM and PM. Each of these areas, however, contains speed-tuned cells that are more likely to be biased to contralateral eye inputs. This greater degree of speed tuning in contralateral dominated neurons may be important for downstream motion processing given our previous findings that direction selectivity is higher for contralateral dominated cells in V1 (Salinas et al., 2017). CMD also eliminated the relationship between ocular dominance and speed tuning in V1 while leaving it intact in LM and PM. This finding suggests that LM and PM may not simply inherit speed-tuning information from V1. In addition, feedback from HVAs to V1 has been shown to influence spatial frequency responses in V1 (Huh et al., 2018). Thus, 
future studies are required to probe whether feedback may contribute to V1 speed tuning and which inputs generate speed tuning in HVAs.

The results of our study and other recently published findings suggest that the response properties of HVAs may depend less on V1 than suggested by the classical hierarchical model. For example, the inactivation of mouse V1 does not alter the spatiotemporal tuning of HVAs, while the inactivation of the superior colliculus (SC) does (Tohmi et al., 2014). The lesion studies of adult SC parallel our findings of depriving visual input during development, as follows: LM shifts to slower speeds and V1 shifts to faster speeds with MD (Tohmi et al., 2014); the areas are less differentiated in velocity tuning. The selective effects we find on speed tuning in V1 hint that speed tuning in LM and PM do not depend solely on V1 input. Moreover, a recent study suggests that a higher visual area called the postrhinal cortex can discriminate motion independently from V1 but requires SC activity (Beltramo and Scanziani, 2019). Eye-specific segregation at the level of SC (Godement et al., 1984; Xu et al., 2011; Seabrook et al., 2017) may provide the driving force for the differentiation of HVAs. Future studies of eye-specific pathways in extrageniculate circuits could help to determine the effects of MD on the extrageniculate pathway and the generation of functionally differentiated HVAs.

\section{References}

Andermann ML, Kerlin AM, Roumis DK, Glickfeld LL, Reid RC (2011) Functional specialization of mouse higher visual cortical areas. Neuron 72:1025-1039.

Beltramo R, Scanziani M (2019) A collicular visual cortex: neocortical space for an ancient midbrain visual structure. Science 363:6469.

Chen T-W, Wardill TJ, Sun Y, Pulver SR, Renninger SL, Baohan A, Schreiter ER, Kerr RA, Orger MB, Jayaraman V, Looger LL, Svoboda K, Kim DS (2013) Ultrasensitive fluorescent proteins for imaging neuronal activity. Nature 499:295-300.

Cruz-Martín A, El-Danaf RN, Osakada F, Sriram B, Dhande OS, Nguyen PL, Callaway EM, Ghosh A, Huberman AD (2014) A dedicated circuit links direction-selective retinal ganglion cells to the primary visual cortex. Nature 507:358-361.

Davis MF, Velez DXF, Guevarra RP, Yang MC, Habeeb M, Carathedathu MC, Gandhi SP (2015) Inhibitory neuron transplantation into adult visual cortex creates a new critical period that rescues impaired vision. Neuron 86:1055-1066.

Dews PB, Wiesel TN (1970) Consequences of monocular deprivation on visual behaviour in kittens. J Physiol 206:437-455.

Dubbs A, Guevara J, Yuste R (2016) moco: fast motion correction for calcium imaging. Front Neuroinform 10:6.

Fagiolini M, Pizzorusso T, Berardi N, Domenici L, Maffei L (1994) Functional postnatal development of the rat primary visual cortex and the role of visual experience: dark rearing and monocular deprivation. Vision Res 34:709-720.

Felleman DJ, Van Essen DC (1991) Distributed hierarchical processing in the primate cerebral cortex. Cereb Cortex 1:1-47.

Gao E, DeAngelis GC, Burkhalter A (2010) Parallel input channels to mouse primary visual cortex. J Neurosci 30:5912-5926.

Garrett ME, Nauhaus I, Marshel JH, Callaway EM (2014) Topography and areal organization of mouse visual cortex. J Neurosci 34:1258712600 .

Glickfeld LL, Andermann ML, Bonin V, Reid RC (2013) Cortico-cortical projections in mouse visual cortex are functionally target specific. Nat Neurosci 16:219-226.

Godement P, Salaün J, Imbert M (1984) Prenatal and postnatal development of retinogeniculate and retinocollicular projections in the mouse. J Comp Neurol 230:552-575.

Gordon JA, Stryker MP (1996) Experience-dependent plasticity of binocular responses in the primary visual cortex of the mouse. J Neurosci 16:32743286.
Heeger DJ, Simoncelli EP, Movshon JA (1996) Computational models of cortical visual processing. Proc Natl Acad Sci U S A 93:623627.

Hess RF, Howell ER (1977) The threshold contrast sensitivity function in strabismic amblyopia: evidence for a two type classification. Vision Res 17:1049-1055.

Hoy JL, Niell CM (2015) Layer-specific refinement of visual cortex function after eye opening in the awake mouse. J Neurosci 35:33703383.

Huh CY, Peach JP, Bennett C, Vega RM, Hestrin S (2018) Feature-specific organization of feedback pathways in mouse visual cortex. Curr Biol 28:114-120.

Huh CYL, Abdelaal K, Salinas KJ, Gu D, Zeitoun J, Figueroa Velez DX, Peach JP, Fowlkes CC, Gandhi SP (2020) Long-term monocular deprivation during juvenile critical period disrupts bino cular integration in mouse visual thalamus. J Neurosci 40:585604.

Jaepel J, Hübener M, Bonhoeffer T, Rose T (2017) Lateral geniculate neurons projecting to primary visual cortex show ocular dominance plasticity in adult mice. Nat Neurosci 20:1708-1714.

Jenks KR, Shepherd JD (2020) Experience-dependent development and maintenance of binocular neurons in the mouse visual cortex. Cell Rep 30:1982-1994.

Ji W, Gămănuţ R, Bista P, D'Souza RD, Wang Q, Burkhalter A (2015) Modularity in the organization of mouse primary visual cortex. Neuron 87:632-643.

Kerlin AM, Andermann ML, Berezovskii VK, Reid RC (2010) Broadly tuned response properties of diverse inhibitory neuron subtypes in mouse visual cortex. Neuron 67:858-871.

Kiorpes L (2006) Visual processing in amblyopia: animal studies. Strabismus 14:3-10.

Kiorpes L, Tang C, Movshon JA (2006) Sensitivity to visual motion in amblyopic macaque monkeys. Vis Neurosci 23:247-256.

Kozma P, Kiorpes L (2003) Contour integration in amblyopic monkeys. Vis Neurosci 20:577-588.

Krahe TE, El-Danaf RN, Dilger EK, Henderson SC, Guido W (2011) Morphologically distinct classes of relay cells exhibit regional preferences in the dorsal lateral geniculate nucleus of the mouse. J Neurosci 31:17437-17448.

Levi DM, Harwerth RS (1977) Spatio-temporal interactions in anisometropic and strabismic amblyopia. Invest Ophthalmol Vis Sci 16:9095.

Livingstone MS, Hubel DH (1987) Psychophysical evidence for separate channels for the perception of form, color, movement, and depth. J Neurosci 7:3416-3468.

Marshel JH, Garrett ME, Nauhaus I, Callaway EM (2011) Functional specialization of seven mouse visual cortical areas. Neuron 72:10401054.

Merigan WH, Maunsell JH (1993) How parallel are the primate visual pathways? Annu Rev Neurosci 16:369-402.

Murakami T, Matsui T, Ohki K (2017) Functional segregation and development of mouse higher visual areas. J Neurosci 37:94249437.

Nassi JJ, Callaway EM (2009) Parallel processing strategies of the primate visual system. Nat Rev Neurosci 10:360-372.

Niell CM, Stryker MP (2008) Highly selective receptive fields in mouse visual cortex. J Neurosci 28:7520-7536.

Prusky GT, Douglas RM (2003) Developmental plasticity of mouse visual acuity. Eur J Neurosci 17:167-173.

Priebe NJ, Lisberger SG, Movshon JA (2006) Tuning for spatiotemporal frequency and speed in directionally selective neurons of macaque striate cortex. J Neurosci 26:2941-2950.

Roth MM, Helmchen F, Kampa BM (2012) Distinct functional properties of primary and posteromedial visual area of mouse neocortex. J Neurosci 32:9716-9726.

Salinas KJ, Velez DXF, Zeitoun JH, Kim H, Gandhi SP (2017) Contralateral bias of high spatial frequency tuning and cardinal direction selectivity in mouse visual cortex. J Neurosci 37:1012510138.

Saravanan V, Berman GJ, Sober SJ (2019) Application of the hierarchical bootstrap to multi-level data in neuroscience. bioRxiv 819334. doi: $10.1101 / 819334$ 
Seabrook TA, Burbridge TJ, Crair MC, Huberman AD (2017) Architecture, function, and assembly of the mouse visual system. Annu Rev Neurosci 40:499-538.

Sereno MI, McDonald CT, Allman JM (1994) Analysis of retinotopic maps in extrastriate cortex. Cereb Cortex 4:601-620.

Simoncelli EP, Heeger DJ (1998) A model of neuronal responses in visual area MT. Vis Res 38:743-761.

Smith IT, Townsend LB, Huh R, Zhu H, Smith SL (2017) Stream-dependent development of higher visual cortical areas. Nat Neurosci 20:200-208

Stephany CÉ, Chan LL, Parivash SN, Dorton HM, Piechowicz M, Qiu S, McGee AW (2014) Plasticity of binocularity and visual acuity are differentially limited by nogo receptor. J Neurosci 34:11631-11640.

Tohmi M, Meguro R, Tsukano H, Hishida R, Shibuki K (2014) The extrageniculate visual pathway generates distinct response properties in the higher visual areas of mice. Curr Biol 24:587-597.
Wang Q, Gao E, Burkhalter A (2011) Gateways of ventral and dorsal streams in mouse visual cortex. J Neurosci 31:1905-1918.

Wang Q, Sporns O, Burkhalter A (2012) Network analysis of corticocortical connections reveals ventral and dorsal processing streams in mouse visual cortex. J Neurosci 32:4386-4399.

Wekselblatt JB, Flister ED, Piscopo DM, Niell CM (2016) Large-scale imaging of cortical dynamics during sensory perception and behavior. J Neurophysiol 115:2852-2866.

Wiesel TN, Hubel DH (1963) Single-cell responses in striate cortex of kittens deprived of vision in one eye. J Neurophysiol 26:1003-1017.

Xu H-p, Furman M, Mineur YS, Chen H, King SL, Zenisek D, Zhou ZJ, Butts DA, Tian N, Picciotto MR, Crair MC (2011) An instructive role for patterned spontaneous retinal activity in mouse visual map development. Neuron 70:1115-1127.

Zhuang J, Ng L, Williams D, Valley M, Li Y, Garrett M, Waters J (2017) An extended retinotopic map of mouse cortex. Elife 6:e18372. 